\title{
Synergistic Modeling of in-vitro and in-vivo data via Stochastic Kriging with Qualitative Factors (SKQ)
}

\author{
Behnam Dehghan
}

Follow this and additional works at: https://researchrepository.wvu.edu/etd

\section{Recommended Citation}

Dehghan, Behnam, "Synergistic Modeling of in-vitro and in-vivo data via Stochastic Kriging with Qualitative Factors (SKQ)" (2016). Graduate Theses, Dissertations, and Problem Reports. 5461.

https://researchrepository.wvu.edu/etd/5461

This Thesis is protected by copyright and/or related rights. It has been brought to you by the The Research Repository @ WVU with permission from the rights-holder(s). You are free to use this Thesis in any way that is permitted by the copyright and related rights legislation that applies to your use. For other uses you must obtain permission from the rights-holder(s) directly, unless additional rights are indicated by a Creative Commons license in the record and/ or on the work itself. This Thesis has been accepted for inclusion in WVU Graduate Theses, Dissertations, and Problem Reports collection by an authorized administrator of The Research Repository @ WVU. For more information, please contact researchrepository@mail.wvu.edu. 


\title{
Synergistic Modeling of in-vitro and in-vivo data via Stochastic Kriging with Qualitative Factors (SKQ)
}

\author{
Behnam Dehghan
}

\author{
Thesis submitted to the \\ Statler College of Engineering and Mineral Resources \\ at West Virginia University \\ in partial fulfillment of the requirements \\ for the degree of
}

Master of Science

in

Industrial Engineering

Feng Yang, Ph.D., Chair

Majid Jaridi, Ph.D.

Robert Mnatsakanov, Ph.D.

Department of Industrial and Management Systems Engineering

\author{
Morgantown, West Virginia \\ 2016
}

Keywords: Stochastic Kriging with Qualitative Factors,

Propranolol matrix extended-release formulations, In-vitro in-vivo

Copyright 2016 Behnam Dehghan 


\section{Abstract \\ Synergistic Modeling of in-vitro and in-vivo data via Stochastic Kriging with Qualitative Factors (SKQ)}

\section{Behnam Dehghan}

The objective of this study is to model in-vivo propranolol plasma concentration after administrating oral propranolol extended-release (ER) tablets. In-vivo data are typically expensive and scarce. To save time and cost needed to achieve high-quality invivo profile, this work utilizes both in-vitro and in-vivo data.

The ensemble of in-vitro and in-vivo data is modeled by stochastic kriging with qualitative factors (SKQ). It treats in-vivo and in-vitro as the two distinct levels of a qualitative factor. By synergistically modeling both types of data, SKQ is able to provide fitted in-vivo profiles whose quality is much higher than those obtained from modeling in-vivo data alone. 


\section{Acknowledgements}

The success of any project depends largely on the advice and encouragement of many others. I take this opportunity to express my gratitude to the people who have been instrumental in the successful completion of this project.

First and foremost I offer my sincerest gratitude to my advisor, Professor Feng Yang, who has supported me throughout my thesis with her patience and knowledge. Words cannot express how grateful I am to her. I feel motivated and encouraged every time I attend a meeting with her.

I also would like to thank, Professor Majid Jaridi and Professor Robert Mnatsakanov for serving on my thesis committee and for their kindness and support.

In addition, I have to thank my friend, Amir Mehdi, who helped me throughout the process of the thesis and gave me his time and suggestions.

Special thanks go to my family, my parents and my brother, for their support and sincere love. 


\section{Table of Contents}

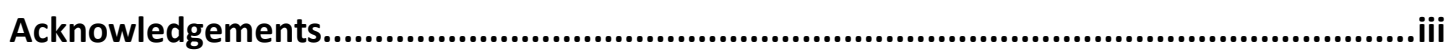

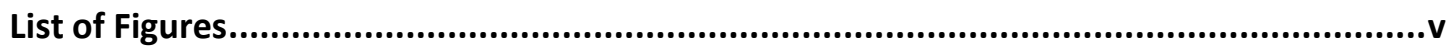

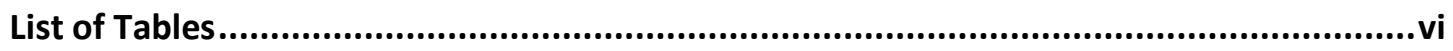

List of Abbreviations ..................................................................................................... vii

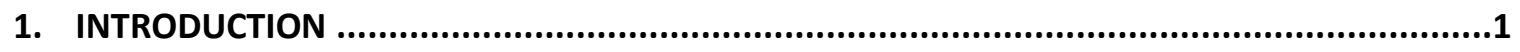

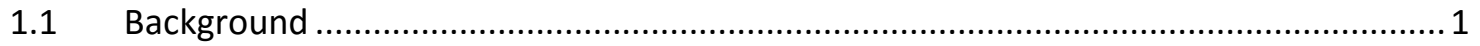

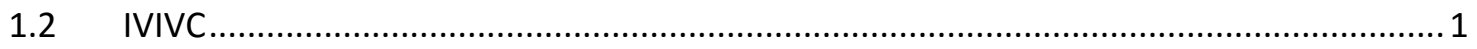

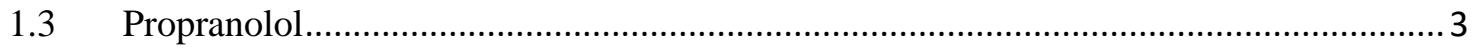

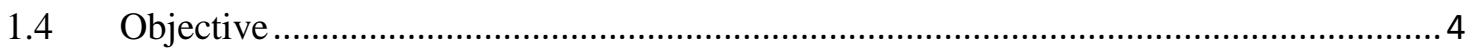

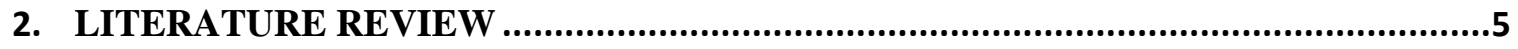

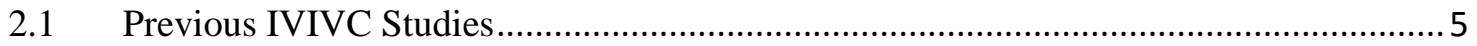

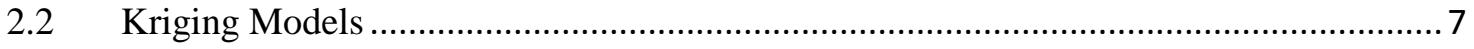

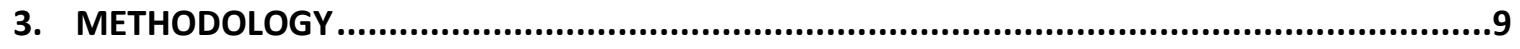

$3.1 \quad$ Review of Stochastic Kriging with Qualitative Factors (SKQ) .................................. 9

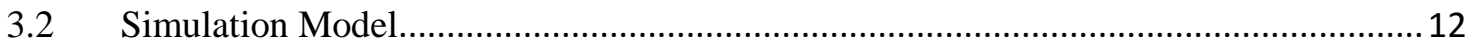

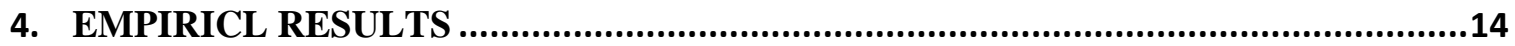

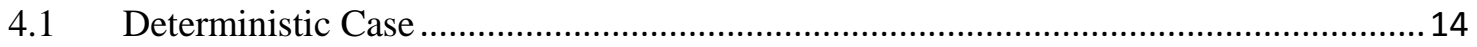

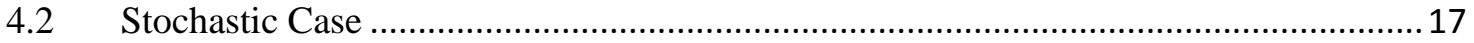

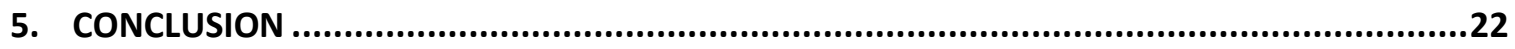

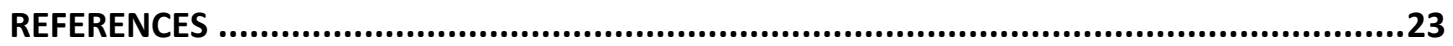

APPENDIX

A Synergistic Modeling of in-vitro and deconvoluted in-vivo data ..................................2

A.1. In-vitro Dissolution Profiles of Propranolol Hydrochloride .....................................27

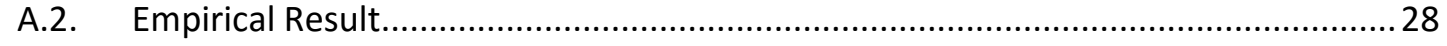

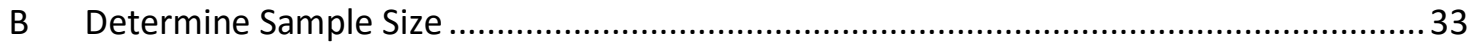

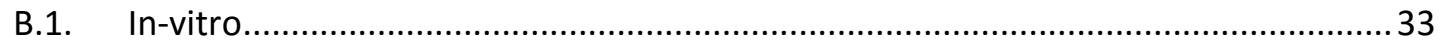

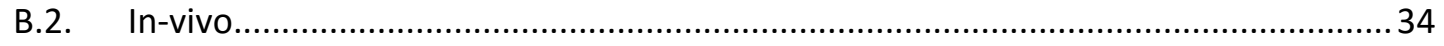




\section{List of Figures}

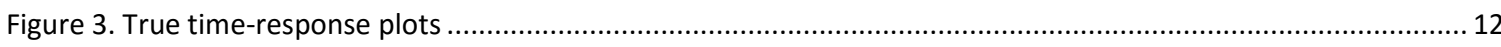

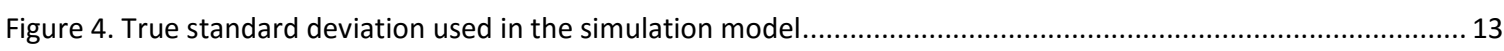

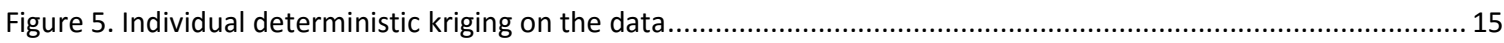

Figure 6. Deterministic kriging with qualitative factors on the data ................................................................. 16

Figure 7. The result of in-vivo DK and DKQ in compare to the in-vivo true response .............................................. 16

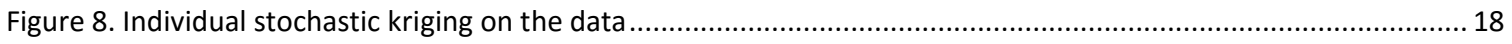

Figure 9. Stochastic kriging with qualitative factors on ERS data ....................................................................... 19

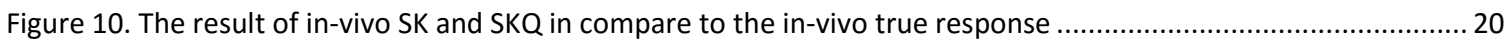

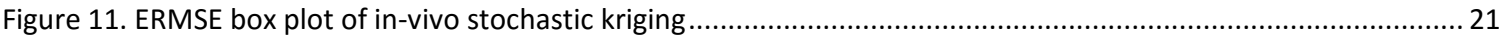

Figure 12. In-vitro dissolution profiles of propranolol hydrochloride from different extended-release tablets............27

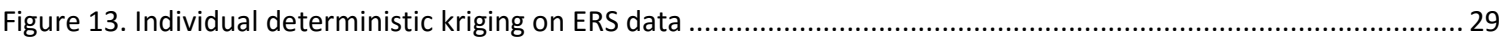

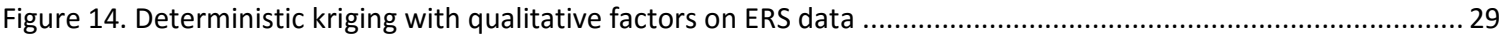

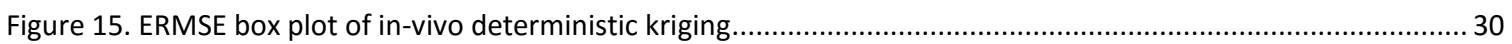

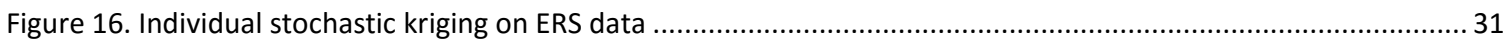

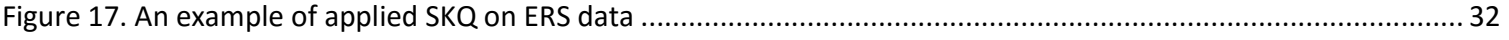

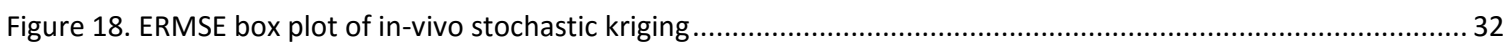




\section{List of Tables}

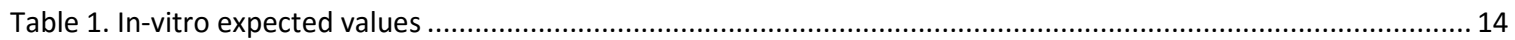

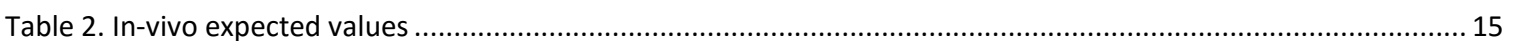

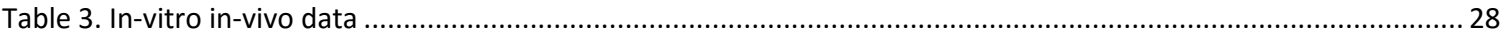




\section{List of Abbreviations}

\begin{tabular}{ll} 
IVIVC & In Vitro In Vivo Correlation \\
FDA & Food and Drug Administration \\
USP & United States Pharmacopeia \\
IR & Immediate Release \\
ER & Extended Release \\
ER-S & Extended Release with a Slow rate of release \\
ER-F & Extended Release with a Fast rate of release \\
DK & Deterministic Kriging \\
DKQ & Deterministic Kriging with Qualitative factors \\
SK & Stochastic Kriging \\
SKQ & Stochastic Kriging with Qualitative factors \\
AUC & Area Under the Curve \\
i.i.d & Independent and Identically Distributed random variables \\
EC & Exchangeable Correlation functions \\
MC & Multiplicative Correlation functions \\
ERMSE & Estimated Root Mean Squared Error \\
\hline
\end{tabular}




\section{CHAPTER 1}

\section{INTRODUCTION}

\subsection{Background}

The development of a new drug takes a lot of cost and time. The expenses can be dramatically reduced if the in-vivo performance of drug formulation can be predicted by in-vitro behavior [35]. In-vitro, which means "within the glass" in Latin, refers to the method of running an experiment on cells or biological molecules outside of a living organism in a controlled environment such as cells in an artificial culture medium. On the other hand, in-vivo, which means "within the living" in Latin, refers to performing an experiment on a whole, living organism such as animal studies and clinical trials [37].

\subsection{IVIVC}

An in-vitro in-vivo correlation (IVIVC) has been defined by the U.S. Food and Drug Administration (FDA) as "a predictive mathematical model describing the relationship between an in vitro property of an extended release dosage form (usually the rate or extent of drug dissolution or release) and a relevant in vivo response, e.g., plasma drug concentration or amount of drug absorbed" [1]. The United States Pharmacopeia (USP) refers to the term in vitro-in vivo correlation as "the establishment of a rational relationship between a biological property, or a parameter derived from a biological property produced by a dosage form, and a physicochemical property or characteristic of the same dosage form" [2]. In-vitro in-vivo correlation (IVIVC) has a significant effect on pharmaceutical development of dosage forms due to its application as a surrogate for in-vivo bioavailability and supporting biowaivers. Since a bioequivalence study is needed to validate a new formulation, which takes a lot of time and money, and as asserted before, time and money are always significant issues. Therefore, in pharmaceutical studies, the application of IVIVC is very important [3].

In order to develop an IVIVC, the observed fraction of the drug absorbed (in-vivo) and that of the drug dissolved (in-vitro) are needed. The observed fraction of the drug 
absorbed can be obtained using the numerical deconvolution method which then will be correlated with in-vitro dissolution data. Performing the deconvolution needs a reference product. The reference product can be an immediate release product or an intravenous solution [1], which in Cheng's et al.[3] study is the commercial immediate-release (IR) tablets (40 mg, AstraZeneca, London, UK). The drug input rate of the dosage form, such as the fraction dose absorbed for oral dosage forms, can be obtained as the result of the deconvolution.

There are four categories of in-vitro in-vivo correlations including: level A, level B, level $\mathrm{C}$, and multiple level C [1]. To develop a level A IVIVC, first the in-vivo plasma concentration data are deconvoluted. Then, the result, the fraction of drug absorbed (deconvoluted in-vivo), with the dissolution data, the fraction of drug dissolved (in-vitro), are used to develop the IVIVC model. The model implies a linear correlation (i.e. a pointto-point relationship) between the fractions of the drug absorbed (deconvoluted in-vivo) and that of the drug dissolved (in-vitro). Based on the obtained IVIVC model, the predicted fraction of the drug absorbed (deconvoluted in-vivo) is calculated from the observed fraction of the drug dissolved (in-vitro). The predicted fraction of the drug absorbed (deconvoluted in-vivo) is then convoluted to the predicted plasma concentrations (in-vivo) [3].

According to Cheng et al.[3], the IVIVC refers to the relationship between in-vitro dissolution profiles of propranolol hydrochloride from different release rates formulations extended-release tablet ER-F (fast rate of release) and ER-S (slow rate of release) and invivo cumulative percentage absorption rate of the same tablets calculated by using the numerical deconvolution approach. One issue about IVIVCs is that they are usually developed in the fasted state [1], like the in-vivo absorption studies performed in the study by Cheng et al.[3] study. In that study, all of the six male Beagle dogs were fasted for twelve hours before the experiment, and they just had access to unlimited water. In some studies, performing the experiment in the fasted state was not possible, thus they try a fed state [1]. Another issue is that in order to obtain the dissolution characteristics of the ER dosage form, the same method should be used for all formulations. In Cheng et al.[3] study, the release characteristic of all the propranolol extended-release tablets were 
determined using USP apparatus I (basket) with a rotation speed of $100 \mathrm{rpm}$ and maintained at $37^{\circ} \mathrm{C}[3]$.

\subsection{Propranolol}

Propranolol is a very effective and non-selective beta-adrenergic medicine which treats high blood pressure, angina (chest pain), irregular heartbeat, migraine headaches, and tremors. Propranolol is also used to improve survival after a heart attack (i.e. it lowers the risk of repeated heart attacks). It works by lowering the blood pressure by changing the transmission of nerve impulses from the brain to the certain part of the body and relaxes blood vessels, which decreases heart rate and pulse. Propranolol is categorized as a beta blockers medication. Beta blockers, which are also known as beta-adrenergic blocking agents, have a variety of usages like reducing blood pressure, and treating glaucoma and migraines. Beta blockers block the effects of the hormone epinephrine (adrenaline) that slows the heartbeat, reduces blood pressure, and improves blood flow [31].

Propranolol is absorbed almost completely from the gastrointestinal tract after oral administration [32], and because of hepatic first-pass effect, its bioavailability is extremely limited, about thirty percent. The hepatic first-pass effect is the drug absorption by the digestive system and entering the hepatic portal system after oral administration, and then goes through the portal vein into the liver. The metabolization of the liver causes only a portion of the drug to go through the circulation system. In other words, the bioavailability of the drug is reduced by liver [31]. To treat high blood pressure, the usual dose of propranolol is 120-240 mg divided in 2-3 doses per day; hence, there is a need to study the once-daily extended-release dosage formulation. Many studies had been reported with respect to controlled-release formulations, while none of them had focused on propranolol matrix ER formulations until Cheng et al. [3] developed an IVIVC model for propranolol matrix ER dosage formulations. 


\subsection{Objective}

The objective of this thesis is to apply stochastic kriging with qualitative factors (SKQ) proposed by Wang et al. [36] to model in-vivo and in-vitro data together for improved fitting of in-vivo behavior. The data used in this work are taken from Cheng et al. [3]. SKQ is to utilize both in-vivo data, which are typically expensive and rare, and in-vitro data, which are usually less expensive and plentiful. By modeling both in-vivo and invitro data together, SKQ-fitted in-vivo profiles are of higher quality than those estimated from in-vivo data alone. Hence, SKQ renders the potential to save money and cost in invivo experiments.

Compared to IVIVC modeling pharmacology, SKQ has certain advantages. As mentioned before, in IVIVC modeling, the numerical deconvolution method is required as the first step. Numerical convolution method is also required as the last step of IVIVC modeling. Each of these steps introduces some additional errors to the model. Whereas, SKQ is free of these complicated convolution/deconvolution steps, and directly models in-vitro and in-vivo data.

The reminder of this thesis is organized as follows. Chapter 2 reviews the relevant literature. Chapter 3 describes the SKQ methodology. In chapter 4, simulation-based case studies are performed and illustrated the advantages of SKQ. 


\section{CHAPTER 2}

\section{LITERATURE REVIEW}

\subsection{Previous IVIVC Studies}

About half a century ago Levy et al. (1965) [4], while working on aspirin tablets found out a substantial correlation between in-vitro dissolution and its in-vivo bioavailability. They developed a single in-vitro dissolution rate test and figured out its correlation with the gastrointestinal absorption of aspirin from three different types of dosage forms. Meanwhile, Wood [5] realized the same fact of high dependency between the drug absorption and dissolution rate. Wagner et al. [6] in 1973 asserted that there is a strong relationship between in-vitro and in-vivo data in an eight-subject two-way crossover study of different digoxin dosage forms. Lindenbaum et al. [7], and Johnson et al. [8] in that year have done the same studies on relationships between in-vitro and in-vivo data of different digoxin dosage forms. After these discoveries, many studies were done about invitro in-vivo correlation (IVIVC) for different drugs and dosage forms. The studies were not limited to animals, such as rats and dogs, but they also included humans in their experiments [9].

The differences in quality of various correlations led to the discovery of different levels of correlation between in-vitro and in-vivo patterns. In level A IVIVC, there is a strong correlation in many researches between dissolution data and drug absorption in the body. The development and internally validation of an in-vitro in-vivo correlation for a hydrophilic matrix extended release metoprolol tablet was done by Eddington ND et al. [10]. A relationship discovered by Emami et al. [11] between in-vivo serum concentration profiles of the adopted sustained-release tablet of LC with a commercial one with the same characteristics in healthy subjects. Royce et al. [12] employed three distinct controlled release formulation principles in formulating 6-N-Cyclohexyl-2-Omethyladenosine to evaluate in-vitro dissolution, in-vivo absorption and their correlation.

On the other hand, many researches demonstrate less strong correlation between dissolution and absorption of a drug than level A, which are classified in Level B. El- 
Yazigi et al. [13] determined the dissolution rates of theophylline from six different commercially available products using the USP and rotating-filter dissolution apparatus to investigate the correlation between in-vitro parameters describing the dissolution characteristics of theophylline dosage forms and the in-vivo parameters. In another study, Dominguez et al. [14] evaluated the bioequivalence of three paracetamol tablets in twelve healthy volunteers using the American innovator product as the reference and they investigated the correlation between in-vitro mean dissolution time from dissolution profiles and in-vivo mean residence time from urinary excretion data.

The other category of a weaker correlation is Level C; such as the study of correlation between dissolution profiles of eleven different brands of phenytoin sodium capsules and observed differences in in-vivo parameters by Shah et al. [15]. In a different study, AlBehaisi et al. [16] established a linear relationship between logarithmic in-vivo blood sampling time and in-vitro dissolution time of deramciclane containing film-coated tablets assigned to equal cumulative area under the curve ratios.

Beside these three categories (levels A, B, and C), some IVIVCs belong to Multiple Level C, which is a powerful correlation and can be as good as Level A. Some studies that belong to this category are mentioned here. Volpato et al. [17] evaluated whether the new condition established by the US Pharmacopeia for the dissolution test would lead to a reasonable correlation between the bioavailability of the two levothyroxine formulations, and this study with levothyroxine tablets was performed on humans for the first time. Lin et al. [18] found the correlation between the in-vivo bioavailability of two different matrix-type aminophylline slow-release tablets in eight healthy male Chinese volunteers and in-vitro dissolution results by moment analysis. Despite the fact that many Level A correlation researches have been done, most of them do not provide appropriate predictability of the correlation assessment. A few numbers of reports include assessing and validation such as the study of correlation between in-vitro dissolution rate done in distilled water with the USP apparatus II and in-vivo after a single-dose administration in the morning by Humbert et al. [19] or the development of an in-vitro in-vivo correlation for two busprione hydrochloride extended-release formulations by Takka et al. [20]. In that study, the validation of IVIVC was determined using prediction errors for Cmax and 
AUC. Although the former was $-0.16 \%$, the latter was $16.1 \%$, which is not acceptable since, based on FDA guidance for Industry Extended Release Oral Dosage Forms [1], an average percent prediction error of less than $10 \%$ for bioavailability parameters is mandatory for all IVIVC studies to prove the reliability of the correlation.

IVIVC can also be used for application of a medicine or drug through the skin (transdermal delivery system) to correlate in-vitro skin permeation data to the in-vivo drug patterns. Qi et al. [21] utilize the convoluted in-vitro skin permeation data of a rabbit to predict the systemic drug concentration of 2,3,5,6-tetramethylpyrazine. The predicted concentrations were correlated well with the observed drug absorption profile. This result proved satisfactory in that the skin permeation tests predict the in-vivo drug profiles.

Fundamentals of the development of study design, lack of the appropriate dissolution design, and the difficulty in the measurement of the drug absorption can be different reasons which prohibit finding correlation between in-vivo parameters and in-vitro drug release data [9]. Siewert [22] classified the role of in-vitro dissolution tests in constructing an IVIVC at three different levels: selection of a drug product, definition of specific in-vitro test system, and provision of comprehensive data and derived parameters with respect to statistical aspects. Prasad et al. [23] show the importance of media selection in construction of IVIVC for quinidine gluconate. They demonstrate the dissolution rates of two different commercial formulations of controlled release $324 \mathrm{mg}$ quinidine gluconate tablets were considerably diverse in water, phosphate buffer $\mathrm{pH} 5.4$, and acetate buffer $\mathrm{pH}$ 5.4.

\subsection{Kriging Models}

Kriging includes a variety of Gaussian process-based modeling methods. Qian et al. [27] developed a general framework for deterministic kriging with qualitative factors, and an iterative estimation procedure for the model is developed. In 2009, a hierarchical Bayesian Gaussian process model was introduced by Han et al. [29]. The model considers both qualitative and quantitative factors assuming that the outputs relating to different levels of a qualitative factor are obtained from Gaussian stochastic processes with similar correlation structures. Zhou et al. [30] keep the flexibility of the unrestrictive 
correlation structure for qualitative factors introduced by Qian et al. [27] while trying to simplify their complex estimation process using the hypersphere parameterization to model the correlation of the qualitative factors. This method deals with standard nonlinear optimization problems with box constraints instead of optimization problems with positive definite constraints. All these methods are for deterministic data, and Wang et al. [36] proposed SKQ, which models stochastic data with both qualitative and quantitative factors. SKQ intends to pool information from all sources of data to obtain the models of the highest quality. 


\section{CHAPTER 3}

\section{METHODOLOGY}

\subsection{Review of Stochastic Kriging with Qualitative Factors (SKQ)}

SKQ is an extension of stochastic kriging (SK). SKQ models the variability of quantitative factors like SK does, but it also considers the variability caused by qualitative factors.

In the following, in-vitro and in-vivo are considered as two different levels of a qualitative factor. Therefore, the experiment is considered as an experiment with one quantitative factor $x$ which is time, and one qualitative factor $z$ with two levels. At design points $w$, we have $\mathbf{w}=(x, z)^{\mathrm{T}}$ with $x \in[0,24]$ hour, and $z \in\left\{c_{1}\right.$ (in-vitro), $c_{2}$ (in-vivo) $\}$.

The response at $\mathbf{w}=(x, z)^{\mathrm{T}}$ on the $\mathrm{n}^{\text {th }}$ replication can be modeled as

$$
y_{\boldsymbol{n}}(\mathbf{w})=\mathrm{Y}(\mathbf{w})+\varepsilon_{n}(\mathbf{w})=\mathbf{f}(\mathbf{w})^{\mathrm{T}} \beta+\mathrm{M}(\mathbf{w})+\varepsilon_{n}(\mathbf{w})
$$

where $\mathbf{f}(\mathbf{w})$ is a vector of known functions and $\beta$ is a vector of unknown parameters that need to be estimated. The term $\mathrm{M}(\mathbf{w})$ represents a realization of a mean zero stationary Gaussian random field. The $\mathrm{M}(\mathbf{w})$ values related to different levels of the qualitative factor are drawn from Gaussian random processes with similar spatial correlation structures and magnitudes of variation. $\varepsilon_{n}(\mathbf{w})$ 's are the i.i.d. mean zero errors for each replication at design point $\mathbf{w}$ [28]. Ankenman et al. [33] refer to $M(\mathbf{w})$ and $\varepsilon_{n}(\mathbf{w})$ as the extrinsic and intrinsic uncertainties at design point $\mathbf{w}$, respectively.

To deal with incorporating the qualitative factors in stochastic kriging, it is necessary to construct a valid spatial correlation functions for $\mathrm{M}$. Wang et al. [36] proposed the following spatial correlation function for $\mathrm{M}$,

$$
\operatorname{Corr}\left[\mathrm{M}\left(\mathbf{w}_{a}\right), \mathrm{M}\left(\mathbf{w}_{b}\right)\right]=\left[\prod_{j=1}^{J} \tau_{j, z_{a j}, z_{b j}}\right] \cdot K\left(\mathbf{x}_{a}, \mathbf{x}_{b}\right)
$$


where $a$ and $b$ are two different design points, and it is assumed the case has $J$ qualitative factors. $\prod_{j=1}^{J} \tau_{j, z_{a j}, z_{b j}}$ corresponding to qualitative factors and the other part, $K\left(\mathbf{x}_{a}, \mathbf{x}_{b}\right)$, dealing with quantitative decision variables. Since in this research, the experiment consists of one qualitative factor, the correlation function is reduced to:

$$
\operatorname{Corr}\left[\mathrm{M}\left(\mathbf{w}_{a}\right), \mathrm{M}\left(\mathbf{w}_{b}\right)\right]=\tau_{z_{a}, z_{b}} \cdot K\left(\mathbf{x}_{a}, \mathbf{x}_{b}\right)
$$

Despite the fact that a variety of different spatial correlation functions for $K\left(\mathbf{x}_{a}, \mathbf{x}_{b}\right)$ exist in the literature, the exponential correlation function is one of appropriate choices

$$
K\left(\mathbf{x}_{a}, \mathbf{x}_{b}\right)=\exp \left\{\sum_{i=1}^{I}-\theta_{i}\left|x_{a i}-x_{b i}\right|^{p}\right\}
$$

where $\theta_{i}$ corresponds to roughness of the response surface and they are not necessarily identical for different coordinates $i=1,2, \ldots, \mathrm{I}$, and such as the previous formula, $a$ and $b$ are two different design points. The parameter $p$ is in $(0,2]$. If $p$ is equal to 2 , the above correlation function would be the popular Gaussian correlation function and the sample paths of a spatial process $M$ are infinitely differentiable. Since in the experiment of this research there is only one quantitative factor, the spatial correlation functions for $K\left(\mathrm{x}_{a}, \mathrm{x}_{b}\right)$ is reduced to

$$
K\left(\mathrm{x}_{a}, \mathrm{x}_{b}\right)=\exp \left\{-\theta\left|x_{a}-x_{b}\right|^{p}\right\}
$$

The other part of spatial correlation function for $\mathrm{M}$, which is $\tau_{z_{a}, z_{b}}$, deals with spatial correlation of qualitative factor. This parameter measure the similarity at any two design points $\mathbf{w}_{a}$ and $\mathbf{w}_{b}$ that differs only on the values of qualitative factor. There are many different correlation functions for the qualitative factors available in the literature that can be used. One of them is isotropic (or exchangeable) correlation functions (EC) that if it is applied in our experiment would be

$$
\begin{aligned}
& \tau_{z_{a}, z_{b}}=\exp \left\{-\emptyset I\left|z_{a} \neq z_{b}\right|\right\} \\
& I\left|z_{a} \neq z_{b}\right|=\left\{\begin{array}{lr}
1 ; & z_{a} \neq z_{b} \\
0 ; & \text { otherwise }
\end{array}\right.
\end{aligned}
$$

where 
The isotropic correlation function assumes that different levels of qualitative factor are of isotropic nature; it means, $\tau_{z_{a}, z_{b}}$ is a constant number, $\exp \{-\emptyset\}$, for all $z_{a} \neq z_{b}$.

Multiplicative correlation function (MC) is another correlation function for qualitative factors that is similar to isotropic correlation functions except that MC assigns different correlation values to different pairs of qualitative factor levels, as one can recognize the difference in the formula which applied to our experiment as follows

$$
\tau_{z_{a}, z_{b}}=\exp \left\{-\left(\emptyset_{a}+\emptyset_{b}\right) I\left|z_{a} \neq z_{b}\right|\right\}
$$

In our case, because the qualitative factor has two levels, it is automatically of an exchangeable nature.

Let $\Sigma_{\varepsilon}$ be the $k \times k$ intrinsic variance-covariance matrix for the vector of averaged errors represented by $\bar{\varepsilon}=\left(\bar{\varepsilon}\left(x_{1}\right), \bar{\varepsilon}\left(x_{2}\right), \ldots, \bar{\varepsilon}\left(x_{k}\right)\right)^{\mathrm{T}}$, where $\bar{\varepsilon}\left(x_{i}\right)=\mathrm{n}_{\mathrm{i}}^{-1} \sum_{j=1}^{n_{i}} \varepsilon_{j}\left(x_{i}\right)$ includes $\mathrm{n}_{\mathrm{i}}$ replications at $x_{i}, i=1,2, \ldots, \mathrm{k}$, assuming there are $\mathrm{k}$ design points. $\Sigma_{\varepsilon}$ can be specified by

$$
\Sigma_{\varepsilon}=\operatorname{diag}\left\{\frac{\sigma_{1}^{2}}{n_{1}}, \frac{\sigma_{2}^{2}}{n_{2}}, \ldots, \frac{\sigma_{k}^{2}}{n_{k}}\right\}
$$

where $\sigma_{i}^{2}=\operatorname{Var}\left[\varepsilon_{j}\left(x_{i}\right)\right]$ is the intrinsic variance at design point $\mathrm{x}_{\mathrm{i}}$. In order to estimate the intrinsic-covariance matrix $\Sigma_{\varepsilon}$, we can use the sample covariance matrix $\hat{\Sigma}_{\varepsilon}$ which its diagonal entries are $\frac{1}{n_{i}\left(n_{i}-1\right)} \sum_{j=1}^{n_{i}}\left(y_{j}\left(\mathrm{x}_{i}\right)-\bar{y}\left(\mathrm{x}_{i}\right)\right)^{2}$ for $i=1,2, \ldots, \mathrm{k}$. This estimation is used in the likelihood function $\mathrm{L}$ as follows

$$
\ln L\left(\delta^{2}, \theta, \Phi\right)=-\frac{1}{2}\left(k \ln (2 \pi)+\ln (|\hat{\Sigma}|)+\tilde{y}^{\mathrm{T}} \hat{\Sigma}^{-1} \tilde{y}\right)
$$

where $|\widehat{\Sigma}|$ is the determinant of $\hat{\Sigma}$ which is equal to $\Sigma_{\mathrm{M}}\left(\delta^{2}, \theta, \Phi\right)+\widehat{\Sigma}_{\varepsilon}$, and $\tilde{y}=\bar{y}-$ $\mathbf{F} \hat{\beta}\left(\delta^{2}, \theta, \Phi\right)$. Matlab's non-linear optimization function, fmincon, takes care of maximizing the log-likelihood function by minimizing $-\ln L$. Subsequently, MLEs for $\delta^{2}, \theta, \Phi$ and $\beta$ can be obtained given $\delta^{2}, \theta, \Phi$ and $\widehat{\Sigma}_{\varepsilon}$, by maximizing likelihood function $\mathrm{L}\left(\delta^{2}, \theta, \Phi\right)$, and the value of $\hat{\beta}\left(\delta^{2}, \theta, \Phi\right)$ would be

$$
\hat{\beta}\left(\delta^{2}, \theta, \Phi\right)=\left(\mathbf{F}^{\mathrm{T}} \hat{\Sigma}^{-1} \mathbf{F}\right)^{-1} \mathbf{F}^{\mathrm{T}} \hat{\Sigma}^{-1} \overline{\mathrm{y}}
$$


where $\mathbf{f}\left(\mathbf{w}_{1}\right)^{\mathrm{T}}, \mathbf{f}\left(\mathbf{w}_{2}\right)^{\mathrm{T}}, \ldots, \mathbf{f}\left(\mathbf{w}_{\mathrm{k}}\right)^{\mathrm{T}}$ are the rows of $k \times p$ matrix $\mathbf{F}$, respectively. In order to perform prediction, Chen et al. [34] proposed the MSE-optimal predictor provided by stochastic kriging as follows

$$
\widehat{\mathrm{Y}}\left(\mathbf{w}_{0}\right)=\mathbf{f}\left(\mathbf{w}_{0}\right)^{\mathrm{T}} \widehat{\boldsymbol{\beta}}+\Sigma_{\mathrm{M}}\left(\mathbf{w}_{0}, .\right)^{\mathrm{T}} \Sigma^{-1}(\overline{\mathrm{y}}-\mathbf{F} \hat{\beta})
$$

Following the corresponding mean squared error of $\widehat{Y}\left(\mathbf{w}_{0}\right)$ is

$$
\operatorname{MSE}\left(\widehat{\mathrm{Y}}\left(\mathbf{w}_{0}\right)\right)=\Sigma_{\mathrm{M}}\left(\mathbf{w}_{0}, \mathbf{w}_{0}\right)-\Sigma_{\mathrm{M}}\left(\mathbf{w}_{0}, .\right)^{\mathrm{T}} \Sigma^{-1} \Sigma_{\mathrm{M}}\left(\mathbf{w}_{0}, . .\right)+\eta^{\mathrm{T}}\left(\mathbf{F}^{\mathrm{T}} \Sigma^{-1} \mathbf{F}\right) \eta
$$

where $\eta=\mathbf{f}\left(\mathbf{w}_{0}\right)-\mathbf{F}^{\mathrm{T}} \Sigma^{-1} \Sigma_{\mathrm{M}}\left(\mathbf{w}_{0},.\right)$.

\subsection{Simulation Model}

The simulation model used to generate simulation data mimicking real experimental data from the study by Cheng et al. [3], is described as follows. The true expected responses for the two subpopulations, in-vitro and in-vivo, respectively represented as $Y\left(x, c_{1}\right)$, $Y\left(x, c_{2}\right)$ :

$$
\begin{aligned}
& Y\left(x, c_{1}\right)=3.5 \times\left(52.26 e^{0.01984 x}-49.24 e^{-0.1889 x}\right)-10.35 \\
& Y\left(x, c_{2}\right)=37182075.0989764 \times e^{-0.22903896 x}-37182073.2671591 e^{-0.2294144 x}-0.0018
\end{aligned}
$$

Figure 3 shows the true time-response plots of propranolol plasma concentration versus time (in-vivo), and propranolol absorption versus time (in-vitro) together.

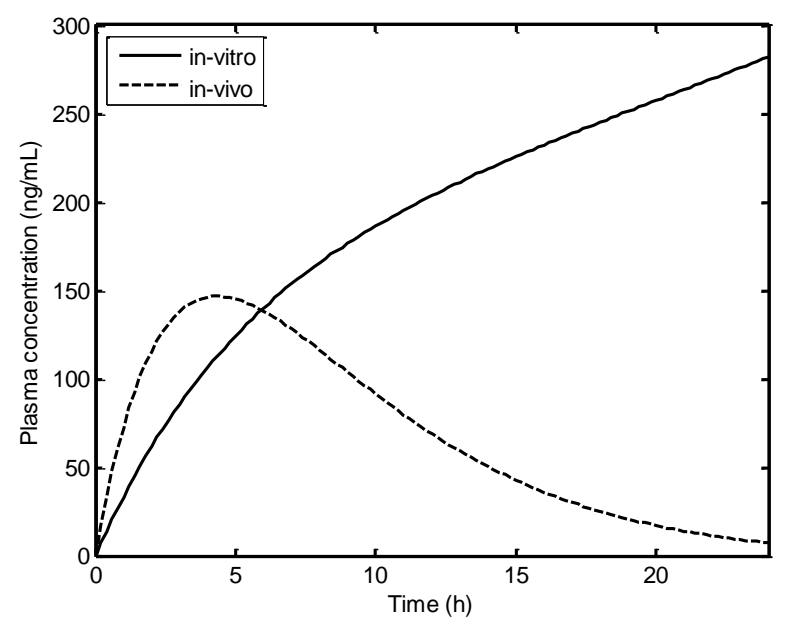

Figure 1. True time-response plots 
Based on Wang et al. [36], the true variance models utilized in the simulation model is described as follows

$$
\begin{array}{ll}
\operatorname{Var}\left[\varepsilon\left(x, c_{1}\right)\right]=\left(0.37 \times \exp \left(Y\left(x, c_{1}\right) \times 0.0068\right)-0.37\right)^{2} ; & \text { in-vitro }(\mathrm{q}=1) \\
\operatorname{Var}\left[\varepsilon\left(x, c_{2}\right)\right]=\left(0.86 \times Y\left(x, c_{2}\right)^{0.41}\right)^{2} ; & \text { in-vivo (q=2) }
\end{array}
$$

For a subpopulation $c_{q}(q=1,2)$ and at time $x_{0}$, a random response $y_{0}$ is simulated as

$$
y=Y\left(x, c_{q}\right)+\sqrt{\operatorname{Var}\left[\varepsilon\left(x, c_{q}\right)\right]} \times \varepsilon ; \quad q=1,2
$$

where $\varepsilon$ is a random error provided by a standard normal random generator. The true standard deviations used in the simulation model are plotted in Figure 4.

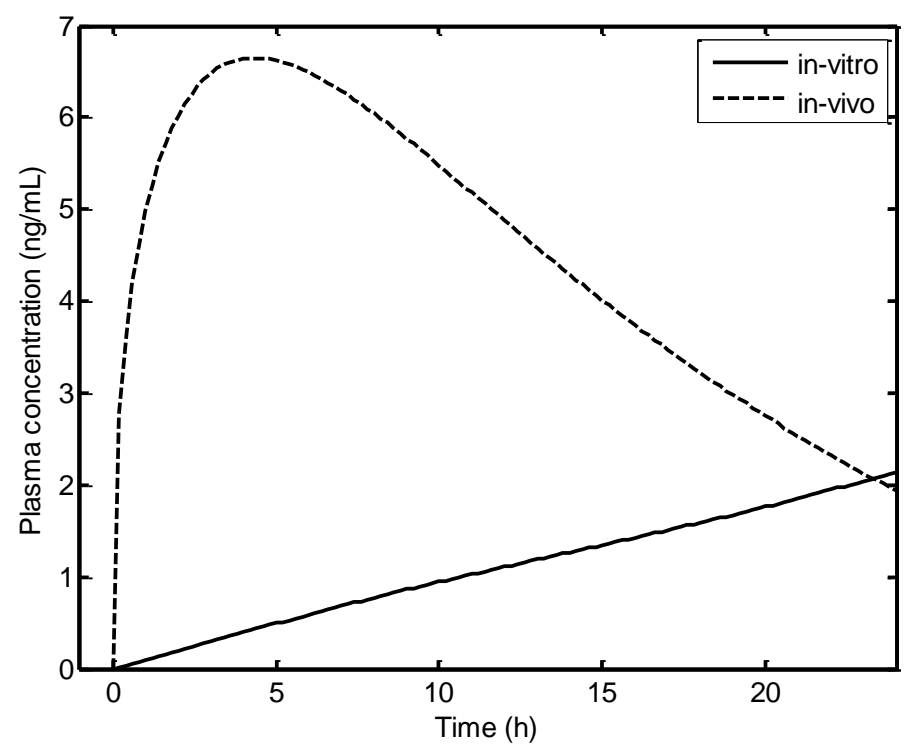

Figure 2. True standard deviation used in the simulation model 


\section{CHAPTER 4}

\section{EMPIRICL RESULTS}

In this thesis, two different cases are considered: deterministic and stochastic. In the former case, deterministic kriging is fitted to the expected values without considering any random errors. Deterministic kriging (DK) and deterministic kriging with qualitative factors (DKQ) are the two methods used in this section. In the latter case, stochastic, 6 replications for in-vitro data and 6 replications for in-vivo data are considered. Stochastic kriging (SK) and stochastic kriging with qualitative factors (SKQ) are the methods taken advantage of in the latter case.

\subsection{Deterministic Case}

It should be noted that in the deterministic approach the random error for the response has not been taken into account. As a result, if the same input is used in the model, the same response will be generated from the model.

For the deterministic case, the true expected responses for each of the two categories are obtained at each of the following equally spaced times

$$
\begin{gathered}
X(\text { in-vitro })=[0,3,6,9,12,15,18,21,24] \text { hours } \\
X(\text { in-vivo })=[0,4,8,12,16,20,24] \text { hours }
\end{gathered}
$$

If $c_{1}$ represents in-vitro and $c_{2}$ represents in-vivo, the random response $y$ is simulated as

$$
\mathrm{y}=\mathrm{Y}\left(\mathrm{x}, \mathrm{c}_{\mathrm{q}}\right) ; \quad \mathrm{q}=1,2
$$

where $\mathrm{Y}\left(\mathrm{x}, \mathrm{c}_{\mathrm{q}}\right)$, described in section 3.2, is the true expected value for subpopulation $\mathrm{q}$ at the abovementioned times.

Table 1. In-vitro expected values

\begin{tabular}{|c|l|c|c|c|c|c|c|c|c|c|}
\hline \multirow{2}{*}{ in-vitro } & Time $(\mathrm{h})$ & 0 & 3 & 6 & 9 & 12 & 15 & 18 & 21 & 24 \\
\cline { 2 - 10 } & Response $(\mathrm{ng} / \mathrm{mL})$ & 0 & 85.77 & 139.98 & 176.62 & 203.65 & 225.61 & 245.10 & 263.62 & 282.04 \\
\hline
\end{tabular}


Table 2. In-vivo expected values

\begin{tabular}{|l|l|c|c|c|c|c|c|c|}
\hline \multirow{2}{*}{ in-vivo } & Time $(\mathrm{h})$ & 0 & 4 & 8 & 12 & 16 & 20 & 24 \\
\cline { 2 - 9 } & Response $(\mathrm{ng} / \mathrm{mL})$ & 0 & 146.09 & 116.23 & 68.96 & 35.91 & 17.04 & 7.22 \\
\hline
\end{tabular}

In-vitro and in-vivo expected values mimicking the real data from Cheng et al. [3] are presented in Table 1 and 2, respectively. Figure 5 shows the result of the DK implementation. In this figure, deterministic kriging is applied to the deterministic (without considering any random error) in-vivo and in-vitro data, separately.

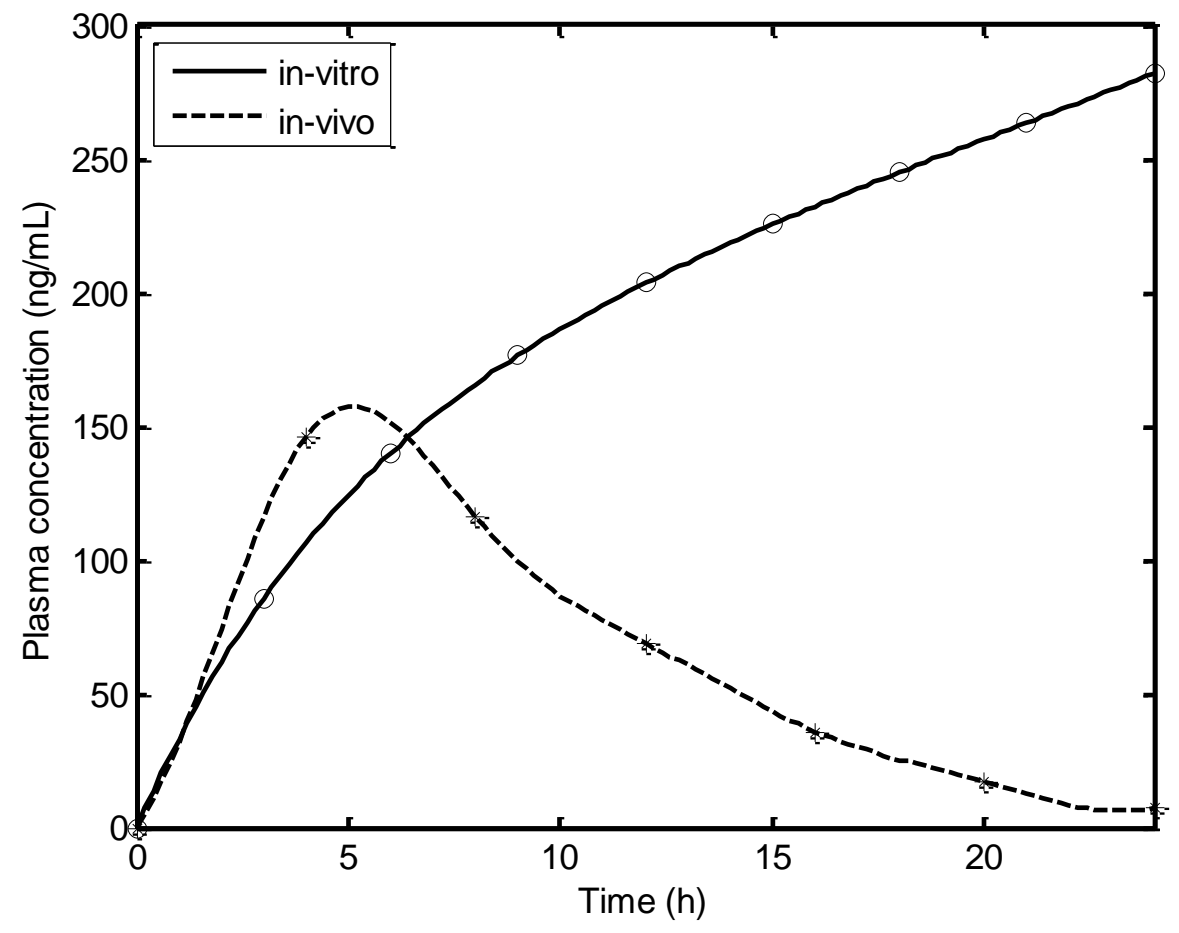

Figure 3. Individual deterministic kriging on the data

Deterministic kriging with qualitative factors (DKQ) is applied to get a better fit. Figure 6 shows the plot of the DKQ implementation. 


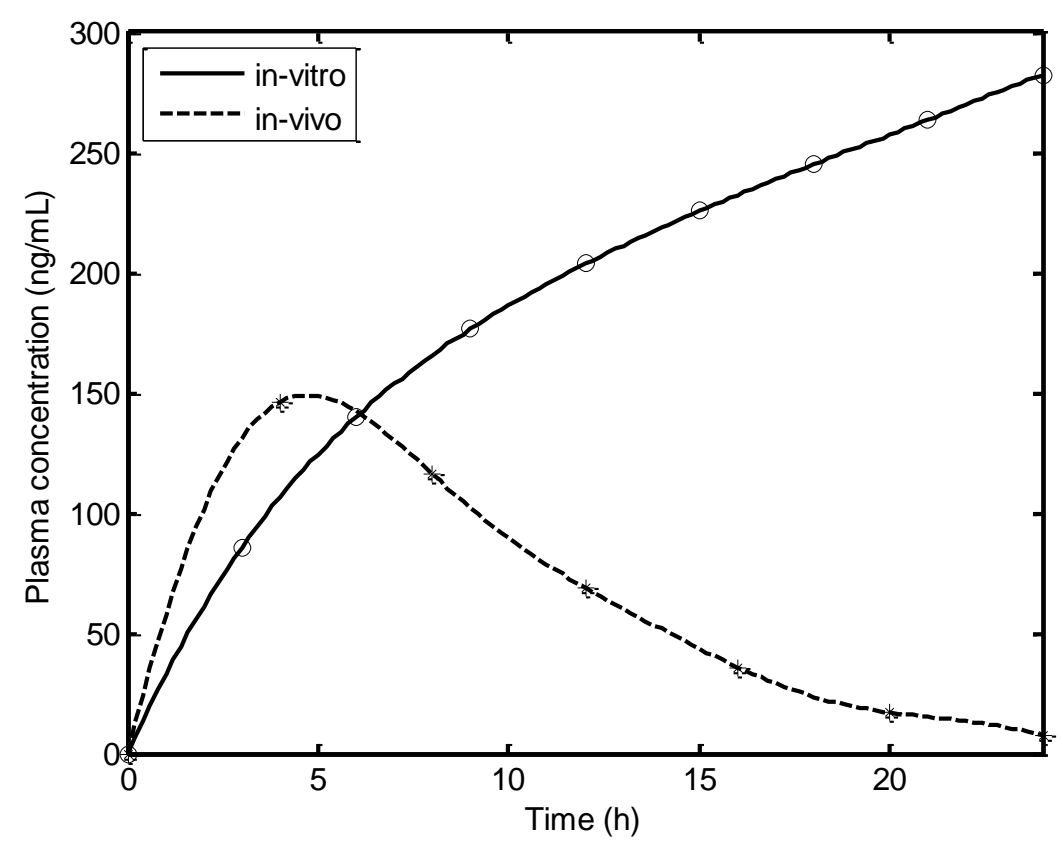

Figure 4. Deterministic kriging with qualitative factors on the data

In order to compare the result of in-vivo DK and DKQ, both of them are plotted in Figure 7 beside the in-vivo true response. As can be seen in the figure, the in-vivo DKQ plot is closer to the true response plot than the DK one.

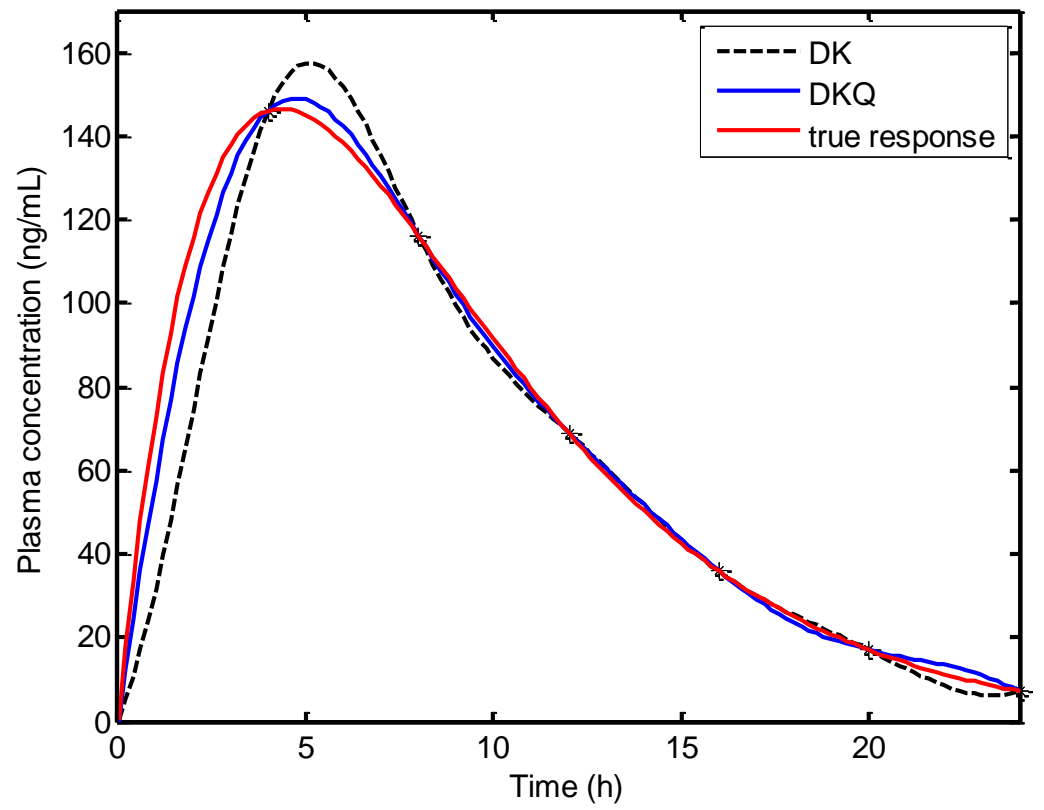

Figure 5. The result of in-vivo $D K$ and $D K Q$ in compare to the in-vivo true response

Besides comparing the result graphically in Figure 7, the estimated root mean squared error can be used. It also helps to evaluate the accuracy of point estimate. 


$$
\operatorname{ERMSE}\left(C_{c_{q}}\right)=\sqrt{\frac{1}{\#\left[C_{\left.c_{q}\right]}\right]} \sum_{\boldsymbol{w} \in C_{c_{q}}}(\hat{Y}(\boldsymbol{w})-Y(\boldsymbol{w}))^{2}}
$$

where \# $\left[C_{c_{q}}\right]$ is the number of check points used. ERMSE measures the average deviation of predicted response from the true response.

To calculate ERMSE for in-vivo deterministic case, the total time range (from 0 to 24 hours) is divided into equally spaced, 0.2-hour, intervals. Therefore, a total number of 121 check points used to calculate the ERMSE, and consequently to evaluate the performances of DK and DKQ in predicting in-vivo response.

ERMSEs are calculated for both "deterministic kriging" and "deterministic kriging with qualitative factors" to compare the methods. ERMSE for DK is equal to 13.41, and the value for DKQ is 4.73. The comparison of these two numbers indicates that the fitted DKQ dominates DK in terms of predicting in-vivo response surface since SKQ has less deviation from the true response than SK.

\subsection{Stochastic Case}

In the real world, the availability of in-vivo data is scarce due to the expense and risks associated with data collection. On the other hand, in-vitro data, which attained much easier and cheaper, can help the fitting to get closer to the true responses.

In this thesis, the in-vitro data is the dissolution amount of propranolol hydrochloride extended-release at 9 equally spaced time points. In-vivo data is the plasma concentration of the observed propranolol from the extended-release (ER) tablets at 7 equally spaced time points. To simulate the data, 6 replications (explained in appendix B) have been considered for in-vitro data and 6 (explained in appendix B) for that of in-vivo at each of the following times in hours for each level of the qualitative factor

$$
\begin{gathered}
X(\text { in-vitro })=[0,3,6,9,12,15,18,21,24] \\
X(\text { in-vivo })=[0,4,8,12,16,20,24]
\end{gathered}
$$


$X=4$ for in-vivo data is a critical point because not only in-vivo data has the largest variance at that point, but also it is the peak of the true response figure. Therefore, 6 more replications are considered for $X=4$ (in-vivo).

If $c_{1}$ represents in-vitro and $c_{2}$ represents in-vivo, the random response $y$ is simulated as

$$
\mathrm{y}=\mathrm{Y}\left(\mathrm{x}, \mathrm{c}_{\mathrm{q}}\right)+\sqrt{\operatorname{Var}\left[\varepsilon\left(x, c_{q}\right)\right]} \times \varepsilon ; \quad \mathrm{q}=1,2
$$

where $\varepsilon$ is a random error provided by a standard normal random generator. The Matlab function, NORMRND(MU,SIGMA), was used to get this job done.

NORMRND(MU,SIGMA) returns an array of random numbers chosen from a normal distribution with mean MU and standard deviation SIGMA. Since it is a standard normal random generator, MU has been considered as zero and SIGMA was set equal to 1 . The other parts, $\mathrm{Y}\left(\mathrm{x}, \mathrm{c}_{\mathrm{q}}\right)$ and $\operatorname{Var}\left[\varepsilon\left(x, c_{q}\right)\right]$, were explained in the methodology chapter, section 3.2.

Figure 8 shows stochastic kriging fitting to the data, separately. In this figure, stochastic kriging is applied to the in-vivo and in-vitro data, separately.

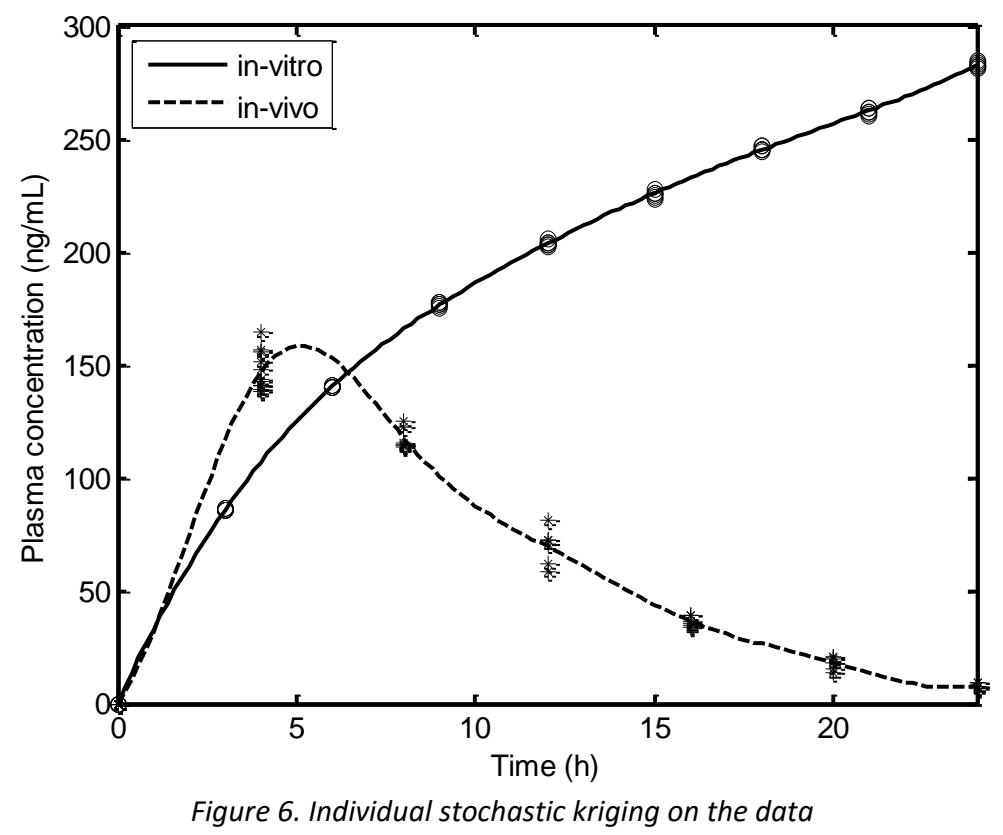

As seen in Figure 8, individual stochastic kriging does not work quite well. Therefore, a need exists to utilize the combined in-vitro and in-vivo data to get a better fit for in-vivo 
behavior. After data from both categories, in-vitro and in-vivo, were combined, the quantitative factor, time (x), and the response $(\mathrm{y})$ were standardized, separately, by the following formula

$$
\text { Standardized data }=\frac{\text { real data }-\min }{\max -\min }
$$

To apply SKQ to the aggregated data, the qualitative factor "study type" was defined; "in-vitro" and "in-vivo" were considered as its two different qualitative factor levels. Then, stochastic kriging with qualitative factors was applied to the standardized data. Figure 9 shows the result of this implementation.

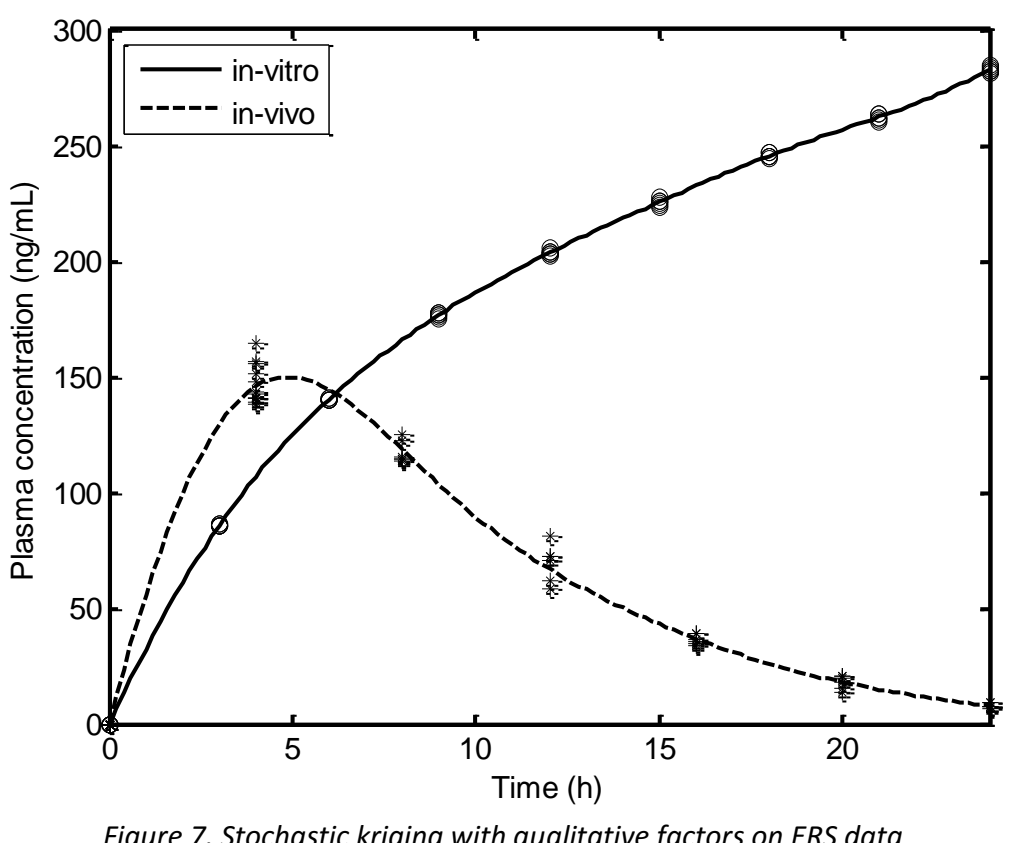

In order to compare the result of in-vivo SK and SKQ, both of them are plotted in Figure 10 beside the in-vivo true response. As can be seen in the figure, the in-vivo SKQ plot is closer to the true response plot than the SK one. 


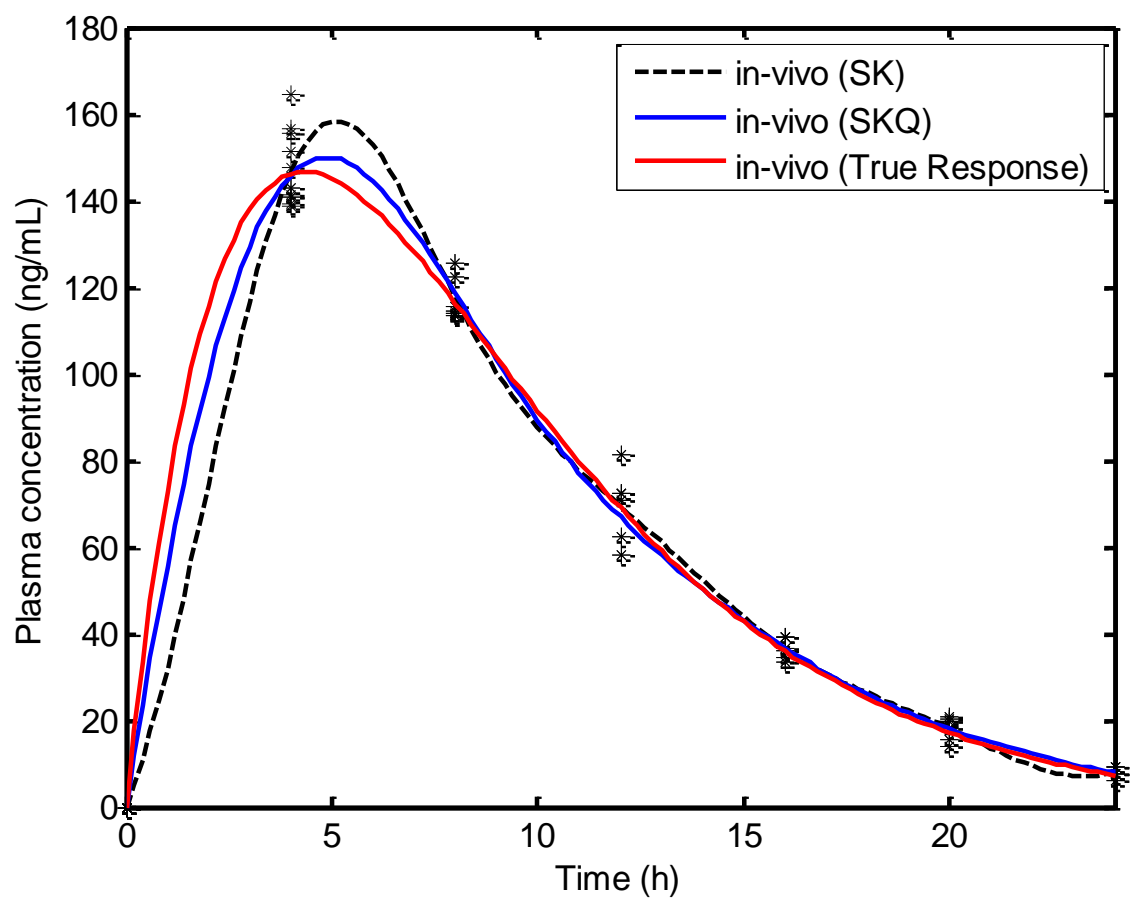

Figure 8. The result of in-vivo SK and SKQ in compare to the in-vivo true response

Besides comparing the result graphically in Figure 10, the estimated root mean squared error can be used. It also helps to evaluate the accuracy of point estimate.

$$
\operatorname{ERMSE}\left(C_{c_{q}}\right)=\sqrt{\frac{1}{\#\left[C_{c_{q}}\right]} \sum_{\boldsymbol{w} \in C_{c_{q}}}(\hat{Y}(\boldsymbol{w})-Y(\boldsymbol{w}))^{2}}
$$

where \# $\left[C_{c_{q}}\right]$ is the number of all check points used. ERMSE measures the average deviation of predicted response from the true response.

To calculate ERMSE for in-vivo stochastic case, like the deterministic case, the total time range (from 0 to 24 hour) is divided into equally spaced, 0.2-hour, intervals. Therefore, a total number of 121 check points used to calculate the ERMSE, and consequently to evaluate the performances of SK and SKQ in predicting in-vivo response.

ERMSEs are calculated for 500 macro-replications of both "individual stochastic kriging" and "stochastic kriging with qualitative factors" to compare the results. Each macro-replication refers to the process of applying SK and SKQ on one randomly generated data-set for both in-vitro and in-vivo using simulation model described in section 3.2. Then ERMSE are calculated for the in-vivo result of each macro-replication. Figure 11 shows the box plot of the calculated ERMSE of the 500 macro-replications. 


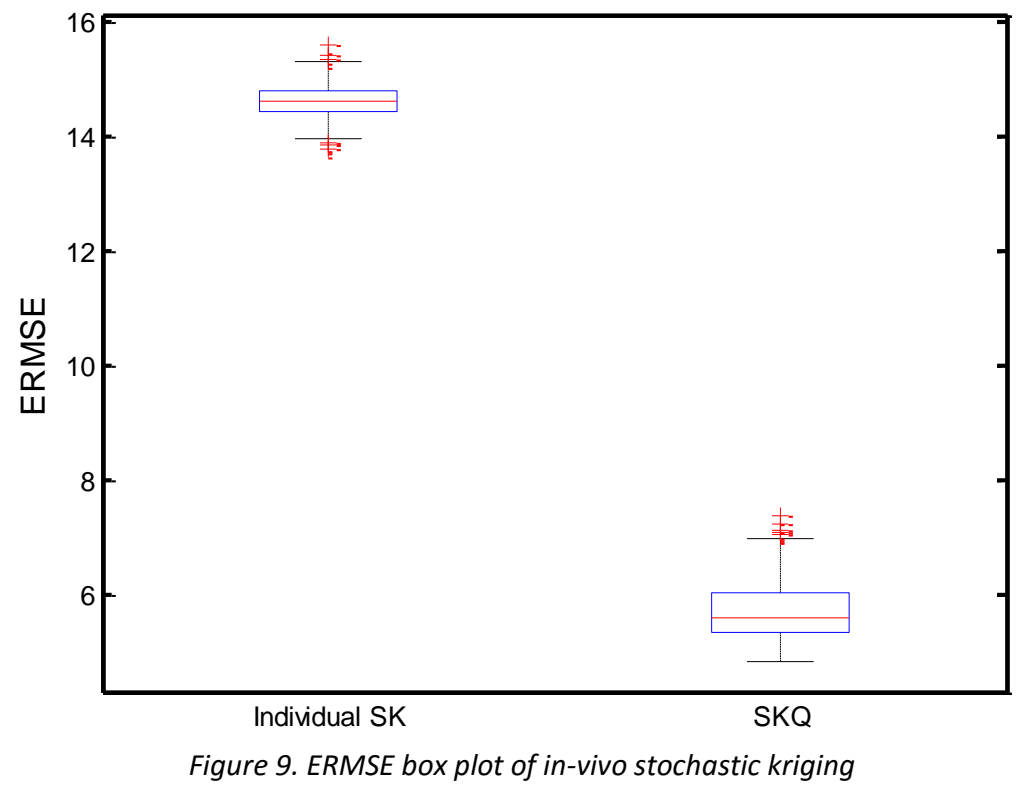

The comparison of the two box plots in Figure 11 indicates that the fitted SKQ performs better than SK because SKQ has less deviation from the true response in all 500 macroreplications. It implies that taking advantage of in-vitro data gives a much better fit of invivo data than applying the model on in-vivo data alone. 


\section{CHAPTER 5}

\section{CONCLUSION}

This thesis applies a metamodeling technique, kriging, to approximate the response of the pharmaceutical in-vivo data (propranolol plasma concentration after administrating oral extended-release formulations). The data was generated by simulation mimicking the real data. Deterministic and stochastic are the two cases that were studied in this thesis.

In the former case, the deterministic case, DK and DKQ were evaluated for the true expected responses. Based on calculated ERMSE, applying the DKQ on deterministic computer experiment provides the in-vivo response closer to the true response than applying DK.

In the latter case, the stochastic case, SK and SKQ were applied to the data considering random errors. SK lacks information pooling ability. Therefore, a separate response SK model is fitted for each data source. On the other hand, SKQ benefits its ability to exploit information from multiple data sources. Therefore, the prediction of in-vivo response can be substantially improved because SKQ allows for the borrowing information from invitro data.

The comparison of SKQ over SK using calculated ERMSE for 500 macro-replications, indicates SKQ gives better result. Consequently, this comparison implies that involving the in-vitro data in the modeling gives a closer fitted model to the true response.

For the future research, a need exists to perform design of experiment (DOE) for both invitro and in-vivo experiments to determine the design points and the number of replications for each of the points. 


\section{REFERENCES}

[1] Guidance for Industry Extended Release Oral Dosage Forms: Development, Evaluation, and Application of In Vitro/In Vivo Correlations, U.S. Department of Health and Human Services, Food and Drug Administration (FDA) Center for Drug Evaluation and Research (CDER), September 1997, BP 2

[2] The United States Pharmacopeia (USP), CHAPTER <1088> IN VITRO AND IN VIVO EVALUATION OF DOSAGE FORMS, USP 29

[3] Cheng, C., Wu, P., Lee, H., Hsu, K. Development and validation of an in vitro-in vivo correlation (IVIVC) model for propranolol hydrochloride extended-release matrix formulations. J. Food and Drug Analysis 22: 257-263, 2014.

[4] Levy, G., Leonards, J.R. and Procknal, J.A. Development of in vitro dissolution tests which correlate quantitatively with dissolution rate-limited drug absorption in man. J. Pharm. Sci. 54: 1719-1722, 1965.

[5] Wood, J.H. In vitro evaluation of physiological availability of compressed tablets. Pharm. Acta Helv. 42: 129-134, 1966.

[6] Wagner, J.G., Christensen, M., Sakmar, E. et al. Equivalence lack in digoxin plasma levels. JAMA, 224: 199-204, 1973.

[7] Lindenbaum, J. and Buttler, V.P. jr., Morphy, J.E., Cresswell, R.M. Correlation of digoxin tablet dissolution-rate with biological availability. Lancet. 1: 1215-1217, 1973.

[8] Johnson, B.F., Greer, H., McCrerie, J. et al. Rate of dissolution of digoxin tablets as a predictor of absorption. Lancet. 1: 1473-1475, 1973.

[9] Jaber Emami. In vitro - In vivo Correlation: Form Theory to Applications. J Pharm Pharmaceut Sci 9: 31-51, 2006

[10] Eddington, N.D., Marroum, P., Uppoor, R. et al. Deveopment and internal validation of an in vitro-in vivo correlation for a hydrophilic metoprolol tarrate extended release tablet formulation. Pharm. Res. 15: 466-473, 1998. 
[11] Emami, J., Tavakoli, N., and Movahedian, A. Formulation of sustained - release lithium carbonate matrix tablets: influence of hydrophilic materials on the release rate and in vitro-in vivo evaluation. J. Pharm. Pharmaceut. Sci. 7: 338-344, 2004.

[12] Royce, A. and Li, S., Weaver, M. and shah, U. In vitro and in vivo evaluation of three controlled release principles of 6-N-cyclohexyl-2'-O-methyladenosine. J. Contrl. Rel. 97: 79-90, 2004.

[13] El-Yazigi, A. and Sawchuk, R.J. In vitro-in vivo correlation and dissolution studies with oral theophylline dosage forms. J. Pharm. Sci. 74: 161-164, 1985.

[14] Dominguez, A., Medina, R., and Hurtado, M. Bioequivalence study of paracetamol tablets: in vitro-in vivo correlation. Drug Dev. Ind. Pharm. 8:821-828, 2000.

[15] Shah, V.P., Prasad, V.K., Alston, T., Cabana, B.E., Gural, R.P., and Meyer, M.C. Phenytoin I: In vitro in vivo correlation for $100-m g$ phenytoin sodium capsules. J. Pharm. Sci. 72 : 307-308, 1983.

[16] Al-Behaisi, S., Antal, I., Morovjan, G., Szunyog, J., Drabant, S., Marton, S., and Klebovich, I. In vitro simulation of food effect on dissolution of deramciclane film-coated tablets and correlation with in vivo data in healthy volunteers. Eur. J. Pharm. Sci. 15: 157-162, 2002.

[17] Volpato, N.M., Silva, R.L., Brito, A.P.P. et al. Multiple level C in vitro/in vivo correlation of dissolution profiles of two 1-thyroxine tablets with pharmacokinetics data obtained from patients treated for hypothyroidism. Eur. J. Pharm. Sci. 21: 655-660, 2004.

[18] Lin, S.Y., Kao, Y.H. and Chang, H.N. Preliminary evaluation of the correlation between in vitro release and in vivo bioavailability of two aminophylline slow-release tablets. J. Pharm. Sci. 79: 326-330, 1990.

[19] Humbert, H., Cabiac, M.D., and Bosshrdt, H. In vitro-in vivo correlation of a modifiedrelease oral form of ketotifen: in vitro dissolution rate specification. J. Pharm. Sci. 83: 131-136, 1994. 
[20] Takka, S., Sakr, A., and Goldberg, A. Development and validation of in vitro-in vivo correlation for buspirone hydrochloride extended release tablets. J. Control. Rel. 88: 147-157, 2003.

[21] Qi, X., Liu, R., Sun, D. et al. Convolution method to predict drug concentration profiles of 2,3,5,6-tetramethylpyrazine following transdermal application. Int. J. Pharm. 259: 39-45, 2003.

[22] Siewert, M. Perspectives of in-vitro dissolution test in establishing in vivo/in vitro correlations. Eur. J. Drug Metab. Pharmacokinet. 18: 7-18, 1993.

[23] Prasad, V., Shah, V.P., and Knight, P. Importance of media selection in establishment of in vitro / in vivo relationship for quinidine gluconate. Int. J. Pharm. 13: 7-13, 1983.

[24] Sacks, J., Schiller, S. B., and Welch, W. J. Designs for Computer Experiments. Technometrics. 31: 41-47, 1989.

[25] Currin, C. Mitchell, T., Morris, M. et al. Bayesian Prediction of Deterministic Functions, With Applications to the Design and Analysis of Computer Experiments. J. American Statistical Association. 86: 953-963, 1991.

[26] Rawlinson, J. J., Furman, B. D., Li, S., Wright, T. M., and Bartel, D. L. Retrieval, Experimental, and Computational Assessment of the Performance of Total Knee Replacement. J. Orthopedic Research. 24: 1384-1394.

[27] Qian, P. Z. G., Wu, H., and Wu, C. F. J. Gaussian Process Models for Computer Experiments With Qualitative and Quantitative Factors. Technometrics. 50: 283-396, 2008.

[28] Chen, X., Wang, K., Yang, F. Stochastic Kriging with Qualitative Factors. Winter Simulation Conference. 790-801, 2013.

[29] Han, G., Santner, T. J., Notz, W. I., and Bartel, D. L. Prediction for Computer Experiments Having Quantitative and Qualitative Input Variables. Technometrics. 51: 278-288, 2009.

[30] Zhou, Q., P. Z. G. Qian, and S. Zhou. A simple approach to emulation for Computer Models with Qualitative and Quantitative factors. Technometrics. 53: 266-273, 2011. 
[31] Gupta, P., Yadav, S. K., et al. A novel graphene and conductive polymer modified pyrolytic graphite sensor for determination of propranolol in biological fluids. Sensors and Actuators B 204: 791-798, 2014.

[32] Partani, P., Modhave, Y., Gurule, S., et al. Simultaneous determination of propranolol and 4-hydroxy propranolol in human plasma by solid phase extraction and liquid chromatography/electrospray tandem mass spectrometry, J. Pharm. Biomed. Anal. 50: 966-976, 2009.

[33] Ankenman, B., Nelson, B. L., and Staum, J. Stochastic Kriging for simulation metamodeling. Operations Research 58: 371-382, 2010.

[34] Chen, X., Ankenman. B. E., and Nelson B. L. The effects of common random numbers on stochastic kriging metamodels. ACM Transactions on Modeling and Computer Simulation 22: 7/1-20, 2012.

[35] Hai Wei. Development of In Vitro/In Vivo Correlations Based on the Biopharmaceutics Drug Classification System. Dissertation. University of Alberta, Spring 2007.

[36] Wang, K., Chen, X., Yang, F., et al. A New Stochastic Kriging Method for Modeling MultiSource Exposure-Response Data in Toxicology Studies. ACS sustainable chemistry \& engineering 2.7 (2014): 1581-1591.

[37] American Psychological Association (2010), "4.21 Use of Italics", The Publication Manual of the American Psychological Association (6th ed.), Washington, DC, USA: APA, ISBN 978-1-4338-0562-2. 


\section{APPENDIX}

\section{A Synergistic Modeling of in-vitro and deconvoluted in-vivo data}

\section{A.1. In-vitro Dissolution Profiles of Propranolol Hydrochloride}

Cheng et al. [3] determined the release characteristics of the propranolol ER tablets using the basket method of USP apparatus I. The temperature was maintained at 98.5 Fahrenheit where the propranolol tablets were kept in $900 \mathrm{~mL}$ of gastric fluid. Samples were collected at different times during 24 hours in batch sizes of five milliliter at a rotation speed of $100 \mathrm{rpm}$. Ultraviolet spectrophotometry at $290 \mathrm{~nm}$ wavelength was used to determine the amount of drug dissolved. Figure 12 illustrates in-vitro dissolution profiles of propranolol hydrochloride. Most of the drug was dissolved after 12 hours. For instance, about eighty percent of ER tablet with a fast rate of release was dissolved after half a day and it was dissolved almost completely within 24 hours.

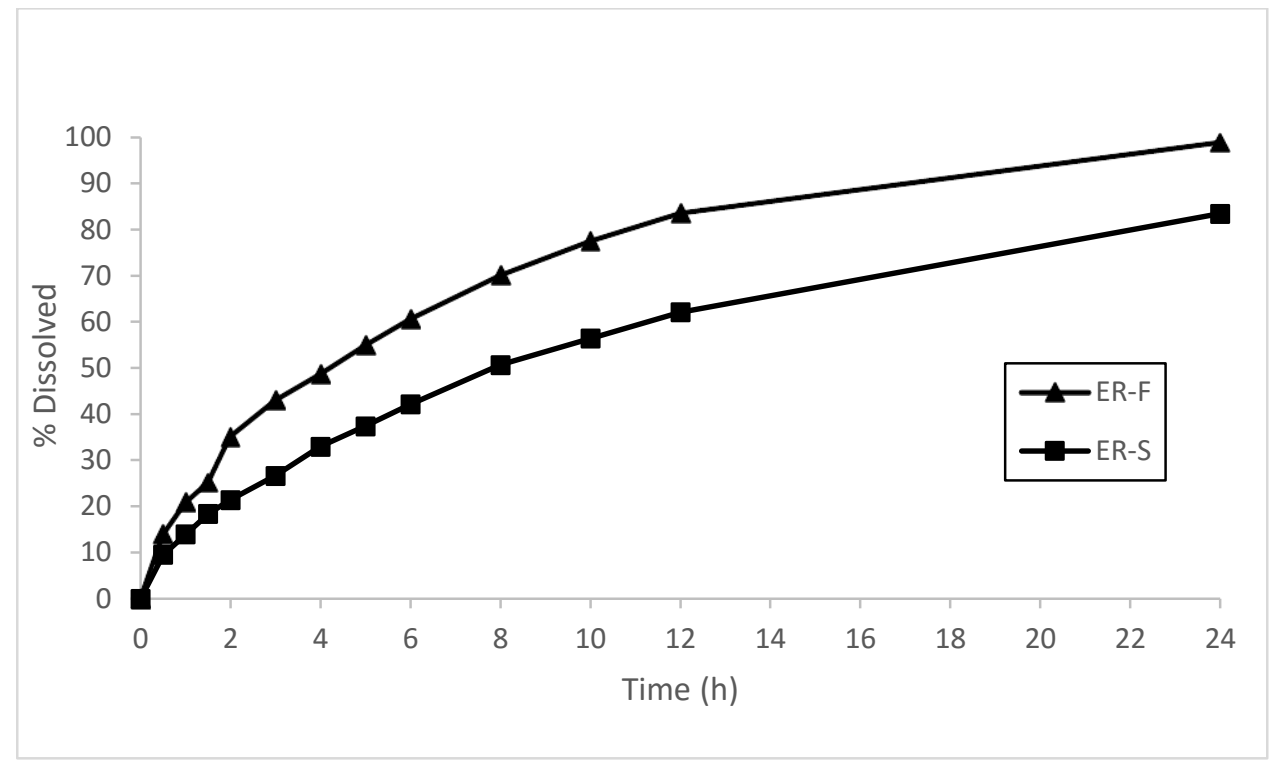

Figure 10. In-vitro dissolution profiles of propranolol hydrochloride from different extended-release tablets

The purpose of this research is to model the mean cumulative percentage absorption rate of extended-release propranolol. Since the data for this work is scarce and expensive to collect, In-vitro dissolution profiles of propranolol hydrochloride data (Figure 12) will be 
taken into consideration as well to get a better model. The goal is pursued using stochastic kriging with qualitative factor proposed by Chen et al. [28].

\section{A.2. Empirical Result}

In this thesis, Matlab was used for two different cases; Deterministic and Stochastic. In the first case, we consider the means as the only points available. Therefore this scenario also can be applied on an experiment without any replication. Individual kriging and kriging with qualitative factors are the two methods used in this section.

In the second case, stochastic, we considered 100 replications for in-vitro data and 6 replications for in-vivo data. Stochastic kriging (SK) and stochastic kriging with qualitative factors (SKQ) are the methods have been taken advantage of.

\section{A.2.1. Deterministic Case}

It should be noted that in the deterministic approach the random error for the response has not been taken into account. As a result, if the same input parameters are used in the model, the same response will be generated from the model in all repeated runs.

Table 3. In-vitro in-vivo data

\begin{tabular}{|c|c|c|}
\hline time $(\mathrm{h})$ & in-vitro & in-vivo \\
\hline 0 & 0 & 0 \\
\hline 0.5 & 5.02 & 5.64 \\
\hline 1 & 9.48 & 12.10 \\
\hline 1.5 & 14.33 & 15.35 \\
\hline 2 & 17.80 & 16.65 \\
\hline 3 & 24.03 & 25.42 \\
\hline 4 & 30.73 & 28.97 \\
\hline 5 & 35.21 & 31.26 \\
\hline 6 & 39.50 & 35.90 \\
\hline 8 & 47.54 & 40.39 \\
\hline 10 & 53.42 & 40.76 \\
\hline 12 & 57.98 & 43.52 \\
\hline 24 & 81.22 & 50.27 \\
\hline
\end{tabular}

Table 1 shows the data obtained from Cheng et al. [3] work. Deterministic kriging is applied on the data. Figure 13 shows the result of the implementation. 


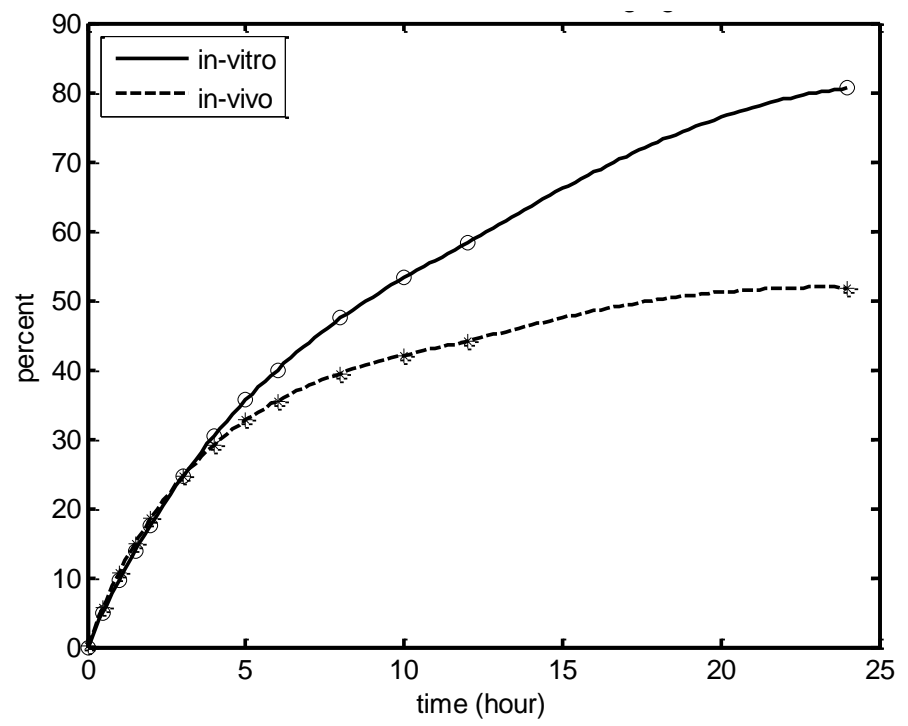

Figure 11. Individual deterministic kriging on ERS data

Deterministic kriging with qualitative factors is applied to get a better fit. Figure 14 shows the plot of the DKQ implementation.

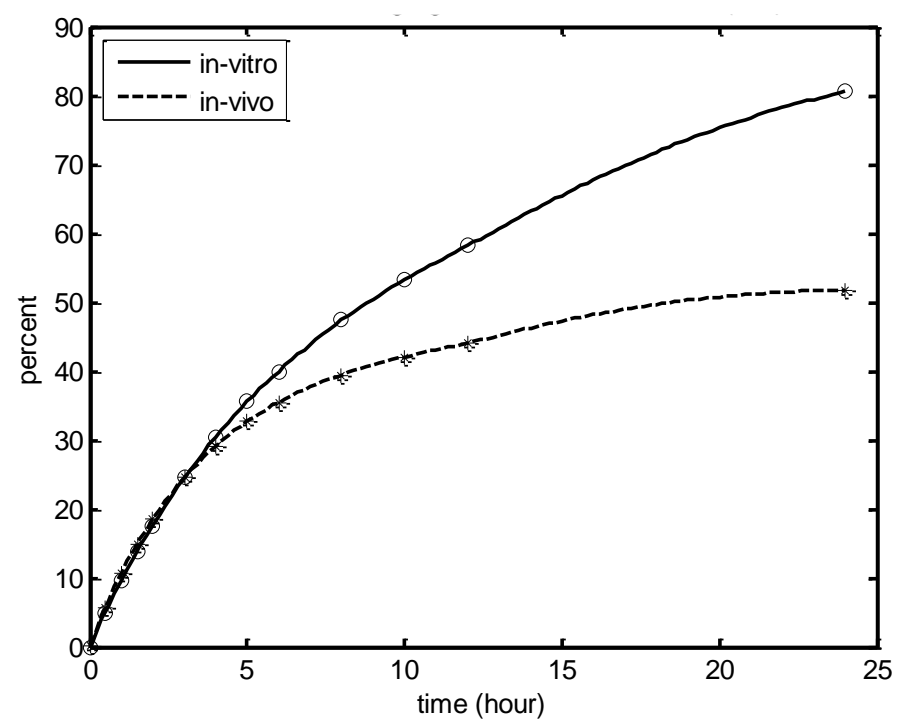

Figure 12. Deterministic kriging with qualitative factors on ERS data

The estimated root mean squared error is calculated to evaluate the accuracy of point estimate. It also helps to compare the applied DK and DKQ.

$$
\operatorname{ERMSE}\left(C_{c_{q}, k}\right)=\sqrt{\frac{1}{\#\left[c_{q}, k\right]} \sum_{w \in C_{c_{q}, k}}(\hat{Y}(w)-Y(w))^{2}}
$$


ERMSEs were calculated in 100 macro-replications for both "individual kriging" and "kriging with qualitative factors" to compare the methods. Figure 15 shows the deterministic in-vivo ERMSE box plot of ERS data.

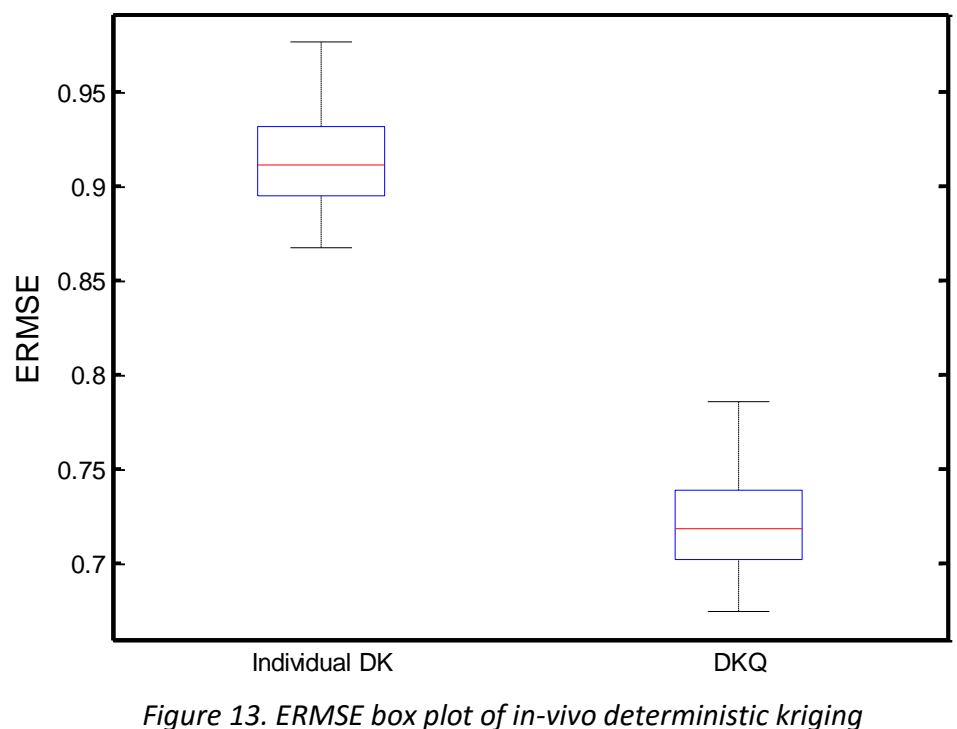

\section{A.2.2. Stochastic Case}

In the real world, the availability of in-vivo data is scarce due to the expense and risks associated with data collection. However, since the in-vitro data are attained much easier and cheaper, a great number of these types of data are accessible. In this thesis, the invitro data is the cumulative percentage of the propranolol hydrochloride extended-release dissolved at different time points. On the other hand, by in-vivo data we mean the cumulative percentage input profiles of propranolol absorbed from the extended-release (ER) tablets. To simulate the data, 100 replications have been considered for in-vitro data and 6 for that of in-vivo at each of these times

$$
X=[0,0.5,1,1.5,2,3,4,5,6,8,10,12,24] .
$$

If $c_{1}$ represents in-vitro and $c_{2}$ represents in-vivo, the random response $y_{0}$ is simulates as

$$
\mathrm{y}_{0}=\mathrm{Y}\left(\mathrm{x}_{0}, \mathrm{c}_{\mathrm{q}}\right)+\varepsilon ; \quad \mathrm{q}=1,2
$$

where $\varepsilon$ is a random error provided by a standard random generator. The Matlab function, NORMRND(MU,SIGMA), was used to get this job done. NORMRND(MU,SIGMA) 
returns an array of random numbers chosen from a normal distribution with mean MU and standard deviation SIGMA. MU has been considered as zero and SIGMA has been equaled to 5 .

After data from both categories, in-vitro and in-vivo, were combined, the quantitative factor, time (x), and the response $(\mathrm{y})$ have been standardized by this formula separately

$$
\text { data_std }=\frac{\text { data }-\min }{\max -\min }
$$

Stochastic kriging was applied on the standardized in-vitro and in-vivo data separately. To apply the stochastic kriging on aggregated data, the qualitative factor "study type" was defined. Hence "in-vitro" and "in-vivo" were considered as its two different qualitative factor levels. Stochastic kriging with qualitative factors was applied on the standardized data.

Figure 20 shows an example of fitting stochastic kriging on ERS data. As in figure 16, individual stochastic kriging does not work quite well in many iterations. So a need is felt to utilize the combined in-vitro and in-vivo data to get a better fit.

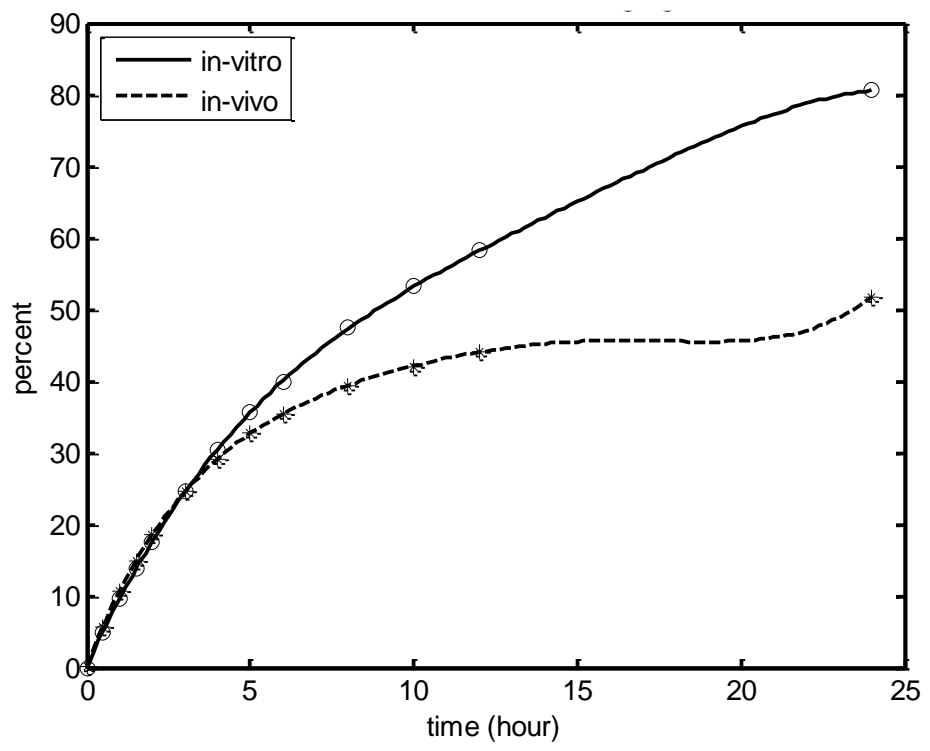

Figure 14. Individual stochastic kriging on ERS data

To apply the stochastic kriging on aggregated data, the qualitative factor "study type" was defined. Hence "in-vitro" and "in-vivo" were considered as its two different 
qualitative factor levels. Stochastic kriging with qualitative factors was applied on the standardized data. Figure 17 shows an example of this implementation.

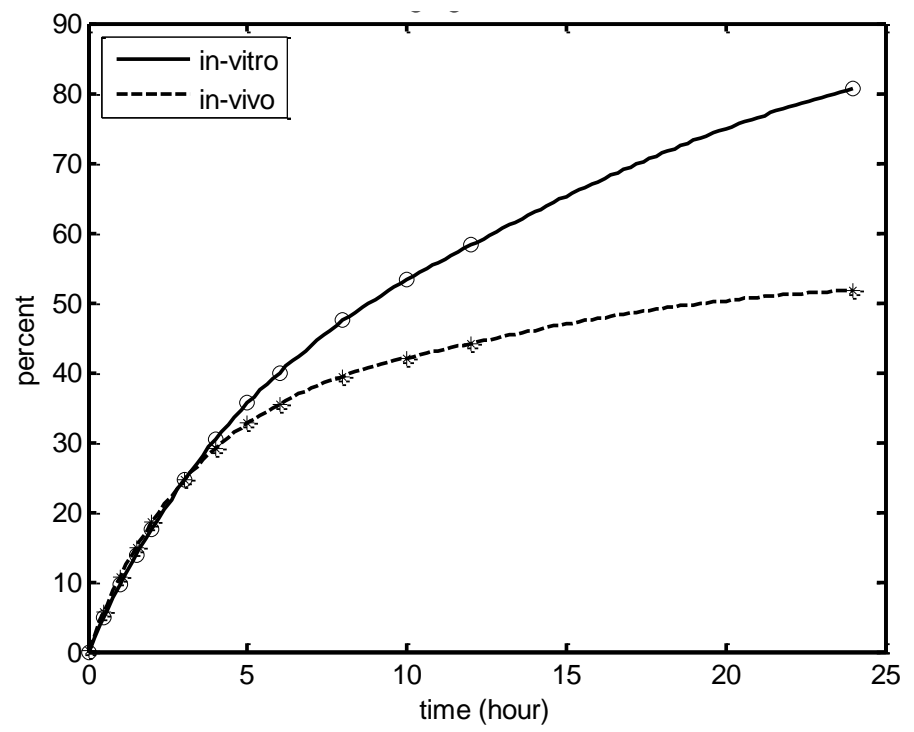

Figure 15. An example of applied SKQ on ERS data

As done in previous section, Deterministic, ERMSEs were calculated in 100 macroreplications for both "individual stochastic kriging" and "stochastic kriging with qualitative factors" to compare them. Figure 18 shows the ERMSE box plot of in-vivo stochastic kriging.

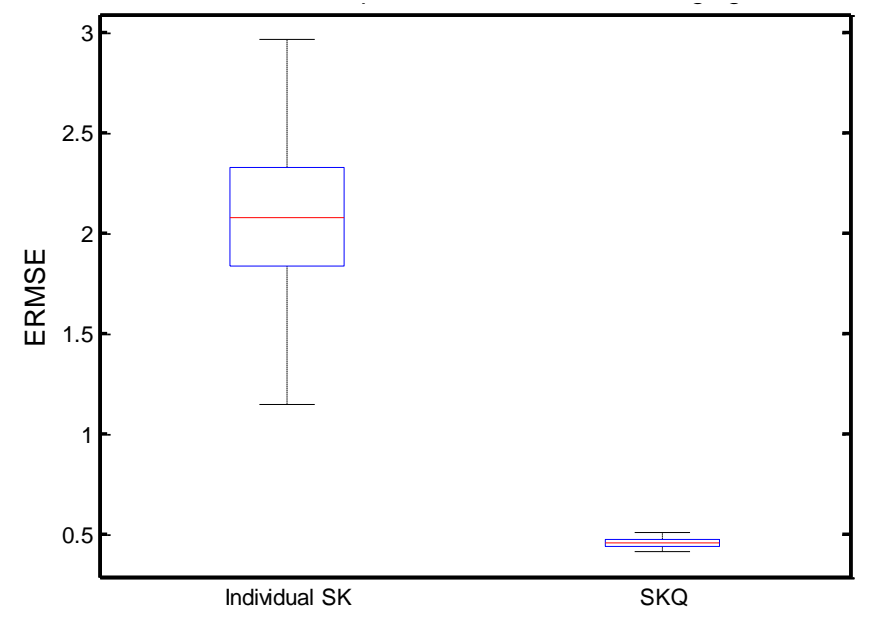

Figure 16. ERMSE box plot of in-vivo stochastic kriging

Figure 18 implies the SKQ works much better than the SK. It means taking advantage of in-vitro data gives a much better fit of in-vivo data than applying the individual stochastic kriging. 


\section{B Determine Sample Size}

\section{B.1. In-vitro}

The desired half-with for in-vitro at $x=24 h$ is considered as $D_{d}=10$. A sample of size $n_{0}=10$ is drawn and $\alpha$ is considered as $5 \%$.

$$
\begin{gathered}
n=\left\lceil\frac{\left(t_{n-1,1-\alpha / 2} \times s\right)^{2}}{D_{d}^{2}}\right\rceil \\
n=\left\lceil\frac{\left(t_{10-1,1-0.05 / 2} \times s\right)^{2}}{100}\right\rceil \\
n=\left\lceil\frac{\left(t_{9,0.975} \times s\right)^{2}}{100}\right\rceil \\
n=\left\lceil\frac{(2.262 \times s)^{2}}{100}\right\rceil
\end{gathered}
$$

The true variance model utilized in the simulation model for in-vitro is

$$
\operatorname{Var}\left[\varepsilon\left(x, c_{1}\right)\right]=\left(0.37 \times \exp \left(Y\left(x, c_{1}\right) \times 0.0068\right)-0.37\right)^{2}
$$

a random response $y_{0}$ is simulated as

$$
y_{0}=Y\left(x_{0}, c_{q}\right)+\sqrt{\operatorname{Var}\left[\varepsilon\left(x_{0}, c_{q}\right)\right]} \times \varepsilon
$$

So, a generated in-vitro sample data at $x=24 h$ would be

\begin{tabular}{|l|l|l|l|l|}
\hline 285.600 & 288.160 & 262.104 & 277.343 & 267.082 \\
\hline 295.391 & 279.348 & 273.837 & 286.908 & 286.934 \\
\hline
\end{tabular}

and $\mathrm{s}$ would be

$$
s=10.341
$$

Then, the sample size required to achieve a $\mathrm{Cl}$ with desired half-width would be

$$
\begin{gathered}
n=\left\lceil\frac{(2.262 \times 10.341)^{2}}{100}\right\rceil \\
n=\lceil 5.4715\rceil=6
\end{gathered}
$$




\section{B.2. In-vivo}

The desired half-with for in-vivo at $x=4.3 h$ is considered as $D_{d}=[5,8]$. A sample of size $n_{0}=$ 6 is drawn and $\alpha$ is considered as $5 \%$.

$$
\begin{gathered}
n=\left\lceil\frac{\left(t_{n-1,1-\alpha / 2} \times s\right)^{2}}{D_{d}^{2}}\right\rceil \\
n=\left\lceil\frac{\left(t_{6-1,1-0.05 / 2} \times s\right)^{2}}{25 \text { or } 64}\right\rceil \\
n=\left\lceil\frac{\left(t_{5,0.975} \times s\right)^{2}}{25 \text { or } 64}\right\rceil \\
n=\left\lceil\frac{(2.570 \times s)^{2}}{25 \text { or } 64}\right\rceil
\end{gathered}
$$

The true variance model utilized in the simulation model for in-vivo is

$$
\operatorname{Var}\left[\varepsilon\left(x, c_{2}\right)\right]=\left(0.86 \times Y\left(x, c_{2}\right)^{0.41}\right)^{2}
$$

a random response $y_{0}$ is simulated as

$$
y_{0}=Y\left(x_{0}, c_{q}\right)+\sqrt{\operatorname{Var}\left[\varepsilon\left(x_{0}, c_{q}\right)\right]} \times \varepsilon
$$

So, a generated in-vivo sample data at $x=24 h$ would be

$$
\begin{array}{|l|l|l|l|l|l|}
\hline 153.751 & 152.997 & 144.993 & 146.013 & 137.805 & 147.975 \\
\hline
\end{array}
$$

and s would be

$$
s=5.8593
$$

Then, the sample size required to achieve a $\mathrm{Cl}$ with desired half-width would be

$$
\begin{gathered}
n=\left\lceil\frac{(2.570 \times 5.8593)^{2}}{25 \text { or } 64}\right\rceil \\
n=\lceil 9.070 \text { or } 3.543\rceil \\
n=4 \text { or } 5 \text { or } 6 \text { or } 7 \text { or } 8 \text { or } 9 \text { or } 10
\end{gathered}
$$


Different numbers of in-vivo data replications from the obtained range, 4 to 10 , are tried. 6replication is selected because the result is stabilized from this point on. 